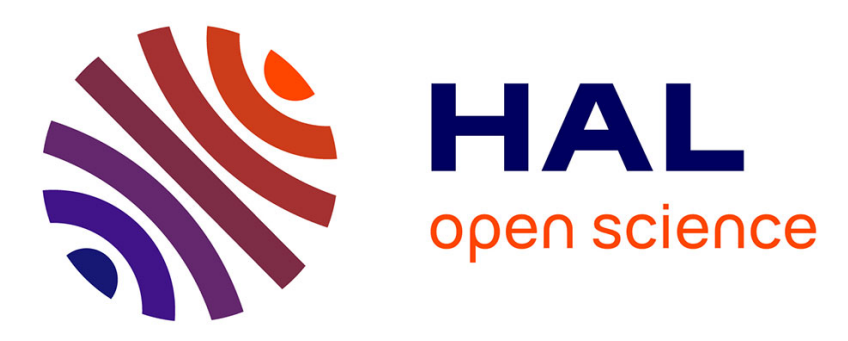

\title{
Particle Coagulation of Emulsion Polymers: A Review of Experimental and Modelling Studies
}

Dang Cheng, Solmaz Ariafar, Nida Sheibat-Othman, Jordan Pohn, Timothy Mckenna

\section{- To cite this version:}

Dang Cheng, Solmaz Ariafar, Nida Sheibat-Othman, Jordan Pohn, Timothy Mckenna. Particle Coagulation of Emulsion Polymers: A Review of Experimental and Modelling Studies. Polymer Reviews, 2018, 58 (4), pp.717-759. 10.1080/15583724.2017.1405979 . hal-02992600

\section{HAL Id: hal-02992600 \\ https://hal.science/hal-02992600}

Submitted on 12 Nov 2020

HAL is a multi-disciplinary open access archive for the deposit and dissemination of scientific research documents, whether they are published or not. The documents may come from teaching and research institutions in France or abroad, or from public or private research centers.
L'archive ouverte pluridisciplinaire HAL, est destinée au dépôt et à la diffusion de documents scientifiques de niveau recherche, publiés ou non, émanant des établissements d'enseignement et de recherche français ou étrangers, des laboratoires publics ou privés. 


\title{
Particle Coagulation of Emulsion Polymers: A Review of Experimental and Modelling Studies
}

Dang Cheng $^{1}$, Solmaz Ariafar ${ }^{1}$, Nida Sheibat-Othman ${ }^{2}$, Jordan Pohn ${ }^{1}$, Timothy F.L. McKenna ${ }^{1}$

1. Université Claude Bernard Lyon 1, CPE Lyon, CNRS, UMR 5265, Laboratoire de Chimie, Catalyse, Polymères et Procédés (C2P2)-LCPP group, Villeurbanne, France

2. Université Claude Bernard Lyon 1, CPE Lyon, CNRS, LAGEP UMR 5007, F-69100, Villeurbanne, France

\begin{abstract}
Particle coagulation, in conjunction with nucleation and growth, plays a significant role in determining the evolution of particle size distribution in emulsion polymerizations. Therefore, many modelling and experimental studies have been carried out to have a better understanding and control of the particle coagulation phenomenon in order to achieve high-quality as well as highly efficient industrial production. This article presents a review of modelling and experimental studies focused on the particle coagulation phenomenon in emulsion polymerizations. The state-of-art of particle coagulation modelling pertaining to emulsion polymerizations is discussed. Experimental studies concerned with latex coagulation processes are summarized next. The review finishes by discussing outstanding problems that need attention and sharing our perspectives on future developments.
\end{abstract}

Keywords: particle coagulation; perikinetic mechanism; orthokinetic mechanism; coagulation model; particle size distribution; emulsion polymerization

\section{Introduction}

Numerous polymeric materials are manufactured by means of emulsion polymerization in the form of colloidal dispersions of nanoparticles (in the size range of $\sim 2 \mathrm{~nm}$ to $1 \mu \mathrm{m}$ ). These products are generally referred to as latexes. Latexes are used in a tremendous variety of modern 
applications including (but not limited to) adhesives, paints, synthetic rubber, textile products, binders and protective coatings. Emulsion polymerization can be used to produce polymers with distinct properties and performance characteristics that can't be realized by other production approaches attributed to its multiphase and compartmentalized nature. Around 13.3 million tons of emulsion polymers (dry basis) were consumed in the year of 2016 with a market value of $\$ 36.4$ billion, and this market is still in full growth (forecast to rise 6.9\% per year through 2016 to 2020) being driven by the need to eliminate volatile organic compounds (VOCs) ${ }^{1}$. Other appealing advantages of the process are convenient control of mass and heat transfer compared to other polymerization techniques (e.g., bulk polymerizations).

One of the key parameters affecting the end-use properties of latex products is the particle size distribution (PSD), including the range of particle size and the desired shape of the PSD. The PSD influences the rheological behavior, maximum solid content, drying characteristics, adhesion, optical properties, film-forming properties and mechanical strength, etc. ${ }^{2}$. It also affects the post-processing of the latexes. For example, fine particles in the reactor outlet might be lost in the downstream recovery process, or may cause troubles in post-processing the recovered resin powder into pellets ${ }^{3}$. It also affects the reaction rate, as the particles represent the reaction site. Knowledge-based control of PSD of latex is therefore essential for high-quality as well as highly efficient industrial productions.

In addition to particle nucleation and particle growth due to the polymerization itself, coagulation is one of the crucial phenomena that determines the evolution of PSD of a product made via emulsion polymerizations ${ }^{4}$. Unexpected particle coagulation can result in the formation of free-floating coagulum or to the fouling of the reactor internals. When it is not desired, coagulum formation not only affects the product quality via the generation of off-spec products, causes loss 
of product, considerably increases reactor downtime, and can also lead to a reduction in the efficiency of heat exchange. Moreover, undesired particle coagulation can also be a major problem in scaling up an emulsion polymerizations from bench scale to industrial reactor. On the other hand, it can also be common practice to provoke well controlled coagulation in order to fine tune the PSD downstream in order to achieve specifically-tailored product properties, especially when one wishes to use the polymer in a dry state.

Researchers from multiple disciplines such as chemistry, physics, fluid dynamics, computational fluid dynamics (CFD) and chemical engineering have contributed to the fundamental understanding of particle coagulation in many disciplines. While there are undoubtedly some commonalities in terms of understanding basic coagulation mechanisms that transcend a particular product or market, some important points will be particular to emulsion polymerization. There are countless publications on this topic from different disciplines, so it is not our intention (nor is it possible) to mention every piece of work that has appeared in the literature; rather we will restrict ourselves to a discussion of the modelling and experimental studies dealing with coagulation as it pertains to emulsion polymerization, and in particular from the perspective of polymer reaction engineering. The main focus here is to sort out the developments in terms of understanding and quantifying the coagulation of emulsion polymers, and to identify some of the outstanding problems that need attention and further consideration from a practical point of view.

Polymer latexes are thermodynamically unstable, and thus will always coagulate - the question is how fast this occurs! The intermolecular forces of attraction cause molecules in the condensed state to cohere ${ }^{2}$, so there is a thermodynamic tendency for the polymer particles to coagulate. Pushed to the extremely this can eventually result in complete phase separation. The aggregation and coalescence of polymer particles reduces the total interfacial area between the particle and the 
aqueous phases, thereby reducing the total Gibbs free energy of the system. Nevertheless, while most polymer latexes are thermodynamically unstable, they are kinetically stable; i.e., if they are properly stabilized, the rate at which the system proceeds towards thermodynamic equilibrium is sufficiently retarded such that it is not uncommon for a polymer latex product to have a shelf life measured in years. The source of this kinetic stability is the presence of a potential energy barrier, which discourages particles from approaching one another closely, and colliding with enough energy to coagulate. The barrier arises from a balance between attractive and repulsive forces; as a general rule, the higher the potential energy barrier, the more stable the latex. With this in mind, events or conditions that give particles enough energy to overcome this energy barrier will inevitably lead to particle coagulation.

The forces of attraction acting between particles in a polymer solution are collectively known as van der Waals forces, which arise from electric dipoles in the atoms. The electric dipoles may be permanent, induced by other molecules or simply a result of fluctuations in the atoms' electron clouds. The forces of repulsion which act between particles can be grouped into four types: (i) electrostatic forces, which depend on the presence of an electric charge at the surface of the particles; (ii) steric forces, which arise from the presence of hydrophilic molecules bound to the surface of the particles; (iii) depletion forces, which arise from the presence of hydrophilic macromolecules dissolved in the aqueous phase; (iv) solvation forces, which arise from the binding of molecules of the dispersion medium to the surface of the particles. Researchers have developed quantitative models for electrostatic and steric stabilization, but the stability conferred through the depletion and solvation forces is difficult to quantify. In particular, depletion stabilization is difficult to differentiate from steric stabilization due to the tendency of hydrophilic molecules that are dissolved in the aqueous media to adsorb on the surface of the particles ${ }^{2}$. It has been proposed that electrostatic and steric repulsive forces are generally more significant ${ }^{5}$. 
There are two mechanisms of coagulation that are most prevelant in case of latex made by emulsion polymerization: perikinetic coagulation and orthokinetic coagulation. Perikinetic coagulation results from the Brownian motion of particles (diffusive forces), while orthokinetic coagulation is facilitated by the motion of the fluid (convective forces). The particles in polymer latex constantly undergo Brownian motion which brings them into intimate proximity with each other. Velocity gradients in the flow field enhance the motion of particles relative to one another, which can increase the force of collision as well as the frequency of collisoins. Of course it is entirely probable that the two mechanisms can coexist in a given system.

Obviously, given its importance, the coagulation of emulsion polymers has been the subject of many mathematical modeling and/or experimental studies during the past decades. Ideally, the experimental studies should shed light on the mechanisms and outcomes of the particle coagulation process, and to help construct physically meaningful mathematical models of the steps involved. Nevertheless, and as we shall see below, given that the phenomena associated particle coagulation of emulsion polymers are clearly quite complex and difficult to quantify à priori, the scientific community has yet to develop the equivalent of a "Grand Unified Theory of Coagulation".

This article is organized as follows. An overview of numerical simulations centering on the PSD evolutions is presented in order to place the fundamental problem in context. This is followed by discussions of model developments of particle coagulation for different mechanisms. After that, relevant experimental investigations are discussed. Finally, discussions on the limitations of the present models and perspectives for future proposals are presented. 


\section{Modeling Particle Coagulation}

One way of understanding the evolution of the particle size distribution (in the absence of nucleation and particle growth) of a latex is with a population balance equation (PBE) as:

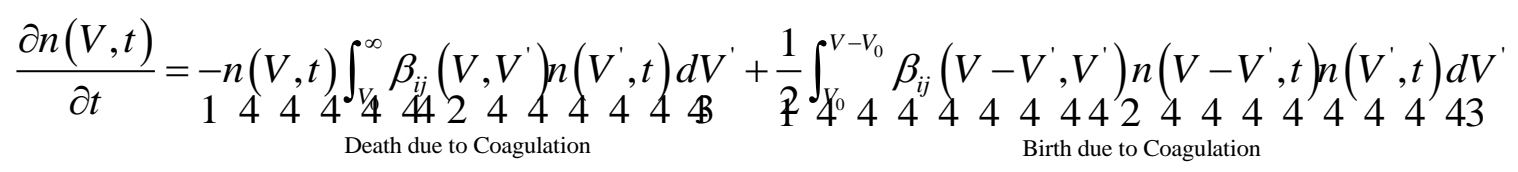

where the PSD is expressed as number density function $n(V, t), \beta_{i j}$ is the coagulation rate coefficient and $V_{0}$ is the minimum size of particles in the system. All the information about physics of particle coagulation process is contained in the coagulation rate coefficient. The term on the left-hand side represents the time accumulation term. The two terms on the right-hand side account for the effect of particle coagulation events. The solution methods of population balance equation can be referred to the books ${ }^{6,7}$.

The coagulation coefficient $\left(\beta_{i j}\right)$ appearing in Eq. (1) is essential for precise description of the evolution of PSD of a latex due to particle coagulation. Efforts have been made to derive coagulation models in a quantitative manner. Smoluchowski ${ }^{8}$ pionneered the analysis of both diffusion- and shear-induced coagulation of non-interacting particles based on the solution of a diffusion or convection-diffusion eqation for the simplified scenario that a particle moving towards a reference particle. In diffusion-induced coagulation, the particle was considered to diffuse towards the reference particle, while he neglected completely the effect of diffusion in the shear-induced coagulation. The boundary conditions adopted were that at the reference particle surface (i.e., the center-to-center separation equals to the sum of their radii), the concentration profile was set to zero as particles that come into contact with the reference particle were assumed to irreversibly aggregate. At an infinite distance from the reference particle, the particle concentration profile was set as its bulk value. The total flux of particles impinging on the 
reference one was defined as the coagulation rate. Fuchs ${ }^{9}$ later analyzed a system in which the interparticle interactions were present, and modified the diffusion equation by means of adding a convective contribution accounting for the hydrodynamics. Together with the Derjaguin-Landau-Verwey-Overbeek (DLVO) theory ${ }^{10}$, the Fuchs-Smoluchowski treatment was ever since popularly used to interpret the colloidal coagulation phenomena.

If all the contributions are taken into account, the generalized Fuchs-Smoluchowski convection-diffusion equation is given by

$$
\nabla \cdot\left(\mathbf{D} \cdot \nabla c-\left(\mathbf{v}_{\text {int }}+\mathbf{v}_{\text {conv }}\right) c\right)=0
$$

where $c$ is particle concentration, $\mathbf{D}$ is the diffusion tensor, $\mathbf{v}_{\text {conv }}$ is the convective velocity and $\mathbf{v}_{\text {int }}$ is the velocity induced by interparticle interactions. In emulsion polymerization reactors, $\mathbf{v}_{\text {conv }}$ acting at the particle scale is usually modeled as an extensional flow field arising from isotropic turbulence as the latex particles are smaller than the Kolmogorov scale. Note that the Eq. (2) was based on the assumption of pair-wise interactions at all levels. More in-depth discussions of this eqaution can be referred to Russel ${ }^{11}$ and Lattuda and Morbidelli ${ }^{12}$.

Three main approaches have been empolyed to investigate the colloidal coagulation in the simultaneous presence of multiple interactions (e.g., diffusion, convection and DLVO interactions) and to understand the complexity of phenomena therein arising. The first one was the trajectory analysis, which solves mathematically simpler problems by completely neglecting diffusive effect (i.e., set $\mathbf{D}=0$ in Eq. (2)). Some useful discoveries have been reported by using this approach, such as stable trajectories for particle pairs under shear due to a balance between shear and van der Waals attractive forces. However, the limitation of this approach is that it underestimates the 
coagulation rate when the Peclet number $(P e)$ is very small because the diffusive effect is neglected.

The second approach is the use of perturbation theory, which comprises mathematical methods for finding asymptotic solutions to Eq. (2) by breaking it into "solvable" and "perturbation" parts. The employment of perturbation methods has evidenced that the diffusion- and shear-induced coagulation was not directly additive. It has been shown that corrections are necessary to account for the influence of shear on diffusion coagulation at small values of Peclet number and of diffusion on the shear coagulation at very large values of Peclet number. The perturbation method can produce insightful results, but it is difficult to quantify the transition between the two asymptotic regimes, namely the intermediate regime of finite Peclet numbers, especially in the presence of repulsive force. This means it is almost impossible to use perturbation method to derive reliable coagulation rate model that is valid for the entire range of Peclet number.

The last approach is the full numerical solution of Eq. (2), which have become popular as computer power soaring in recent years. Several attempts have been made to numerically solve Eq. (2) for different cases in order to derive coagulation model that is valid for large range of Peclet number. Zinchenko and Davis ${ }^{13,14}$ numerically solved Eq. (2) for the case of fluid droplets for arbitrary values of Peclet number, but in the presence only of attractive forces. After transforming the elliptic Eq. (2) into a parabolic form by neglecting diffusion in the tangential direction, Melis et al. ${ }^{15}$ and Baldyga et al. ${ }^{16}$ numerically solved it for particles experiencing extensional flow. Their calculations illustrated that the transition between shear coagulation and diffusion coagulation is 
smooth in the absence of a repulsive force, while the transition is sharp in the presence of repulsive interactions. Using the same approach, Lattuada and Morbidelli ${ }^{12}$ carried out a systematic investigation of the effect of electrostatic repulsive interactions on the coagulation rate of colloidal partilces in the presence of a convective transport mechanism. They attempted to derive expressions for coagulation rate that can cover the entire range of Peclet number.

By choosing a suitable coagulation model, Eq. (1) can be applied to various applications. Indeed, particle coagulation merely is one of the many complex mechanisms exsting in emulsion polymerizations ${ }^{17,18}$. However, its significance can't be underestimated due to the strong coupling among nucleation, particle growth and particle coagulation phenomena, both when the surfactant concentrations are below and above the CMC ${ }^{19-21}$. The varying stabilization conditions should be accounted for during the emulsion polymerization as reflected by the increasing latex particle size, and the distribution of particles over a range of sizes ${ }^{2,22}$. In order to present how particle coagulation was dealt with in such applications, an overview of the modelling studies of full PSD incorporating particle coagulation of emulsion polymerizations during the past decades is summarized in Table 1. It is worth noting that the comprehensive models incorporating nuclealtion, growth and coagulation phenomena generally have shown satisfactory accord with corresponding experiments for a wide range of systems and operating conditions 4, 23-35. Immanuel et al. ${ }^{27}$ and Fortuny et al. ${ }^{29}$ in particular have quantitatively examined the influence of coagulation events during polymerizations on the final PSD and total particle numbers. They highlighted that coagulation leads to a smaller number of particles in the final latexes, simulations results would be deviated from the experimental data to a large extent if the particle coagulation phenomena was not taken into account in the PSD modellings.

Table 1. Full PSD modelling studies incorporating particle coagulation. 
(N, G, C signify nucleation, growth and coagulation, respetively)

\begin{tabular}{|c|c|c|c|c|c|c|}
\hline Refs & Reactor & Monomers & Surfactant & Initiator & Mechanisms & $\begin{array}{l}\text { Coagulation } \\
\text { Model }\end{array}$ \\
\hline 23 & CSTR & $\begin{array}{l}\text { Sty/ } \\
\text { MMA }\end{array}$ & Anionic & APS & $\mathrm{N}, \mathrm{G}, \mathrm{C}$ & Peri- \\
\hline 24 & Semibatch & $\begin{array}{l}\text { MMA/ } \\
\text { BuA }\end{array}$ & $\begin{array}{l}\text { Anionic/ } \\
\text { Non-ionic }\end{array}$ & APS & $\mathrm{N}, \mathrm{G}, \mathrm{C}$ & Peri- \\
\hline 36 & Semibatch & $\mathrm{VAc} / \mathrm{BuA}$ & Non-ionic & APS & $\mathrm{G}, \mathrm{C}$ & Peri- \\
\hline 37 & Batch & VAc & Anionic & - & $\mathrm{C}$ & Peri- \\
\hline 25 & $\begin{array}{l}\text { Loop } \\
\text { Reactor }\end{array}$ & $\mathrm{VAc} / \mathrm{BuA}$ & $\begin{array}{l}\text { Anionic/ } \\
\text { Non-ionic }\end{array}$ & $\begin{array}{l}\text { KPS- } \\
\text { NaPS }\end{array}$ & $\mathrm{N}, \mathrm{G}, \mathrm{C}$ & Peri- \\
\hline 26 & Semibatch & Sty & Anionic & APS & $\mathrm{N}, \mathrm{G}, \mathrm{C}$ & Peri- \\
\hline 38,39 & Semibatch & $\mathrm{VAc} / \mathrm{BuA}$ & Nonionic & $\begin{array}{l}\text { tBHP- } \\
\text { SFS }\end{array}$ & $\mathrm{N}, \mathrm{G}, \mathrm{C}$ & Peri- \\
\hline 28 & Batch & Sty/EHA & Nonionic & APS & $\mathrm{N}, \mathrm{G}, \mathrm{C}$ & Peri- \\
\hline 40 & Batch & BuA/MMA & Anionic & APS & $\mathrm{N}, \mathrm{G}, \mathrm{C}$ & Peri- \\
\hline 29 & Batch & BuA/MMA & Anionic & APS & $\mathrm{N}, \mathrm{G}, \mathrm{C}$ & Peri- \\
\hline 30,31 & Batch & VAc & $\begin{array}{l}\text { Anionic/ } \\
\text { Non-ionic }\end{array}$ & APS & $\mathrm{N}, \mathrm{G}, \mathrm{C}$ & Peri- \\
\hline 4 & Batch & $\mathrm{VC}$ & Anionic & APS & $\mathrm{N}, \mathrm{G}, \mathrm{C}$ & Peri- \\
\hline 41 & Semibatch & Sty & Anionic & - & $\mathrm{C}$ & Peri- \\
\hline 32 & Batch & Sty & $\begin{array}{l}\text { Anionic/ } \\
\text { Non-ionic }\end{array}$ & KPS & $\mathrm{N}, \mathrm{G}, \mathrm{C}$ & Peri- \\
\hline 33 & Semibatch & BuA/MMA & $\begin{array}{l}\text { Anionic/ } \\
\text { Nonionic }\end{array}$ & KPS & $\mathrm{N}, \mathrm{G}, \mathrm{C}$ & Peri- \\
\hline 34 & Semibatch & VDF & Anionic & KPS & $\mathrm{N}, \mathrm{G}, \mathrm{C}$ & Peri- \\
\hline 42 & Batch & VDF & Anionic & - & $\mathrm{C}$ & Peri- \\
\hline 43 & Batch & $\mathrm{VC} / \mathrm{EA}$ & Nonionic & APS & $\mathrm{G}, \mathrm{C}$ & Ortho- \\
\hline 44 & Semibatch & Sty/But/AA & Anionic & APS & $\mathrm{G}, \mathrm{C}$ & Ortho- \\
\hline 45 & Batch & Sty & Nonionic & - & $\mathrm{C}$ & Ortho- \\
\hline 46,47 & Semibatch & MMA/BuA & Anionic & APS & $\mathrm{G}, \mathrm{C}$ & Ortho- \\
\hline 48 & Batch & Sty & Anionic & APS & $\mathrm{G}, \mathrm{C}$ & Ortho- \\
\hline 3 & Batch & $\mathrm{ABS}$ & Anionic & - & $\mathrm{C}$ & Ortho- \\
\hline 49 & Batch & MMA & Anionic & - & $\mathrm{C}$ & Ortho- \\
\hline 50 & Batch & Sty/Ac & - & - & $\mathrm{C}$ & Combined- \\
\hline 51 & Batch & $\mathrm{VC} / \mathrm{EA}$ & Nonionic & - & $\mathrm{C}$ & Combined- \\
\hline 52 & Semibatch & VDC & Anionic & - & $\mathrm{C}$ & Combined- \\
\hline 35 & Batch & $\mathrm{VC}$ & Anionic & APS & $\mathrm{N}, \mathrm{G}, \mathrm{C}$ & Combined- \\
\hline 53 & Batch & VDF & Anionic & APS & $\mathrm{C}$ & Combined- \\
\hline
\end{tabular}

In terms of coagulation modelling, many researchers have assumed perikinetic coagulation was the dominant mechanism while neglected orthokinetic effect when modelling emulsion polymerization applications as shown in Table 1. This simplification was sometimes justified 
based on experimental results ${ }^{35,37}$, however theoretical studies of particle coagulation suggested that hydrodynamics can play an important role when ionic strength is low and the double layer is thick ${ }^{15}$. In practical emulsion polymerization systems, latex particles tend to collide driven by both Brownian and fluid motion. Theoretically, the presence of shear complicates the modelling of particle coagulation and demands much more complex mathematical treatment. Therefore, strictly speaking, it is of considerable importance to determine the relative magnitude of orthokinetic coagulation to perikinetic coagulation for modelling purposes, given that pure Brownian mechanism is mathematically easier to handle. The ratio is characterized by Peclet number as ${ }^{54,55}$

$$
P e=\frac{3 \pi \mu G r_{i} r_{j}\left(r_{i}+r_{j}\right)}{2 k_{B} T}
$$

where $G$ is the shear rate. When $P e<<1$, the coagulation can be reasonably approximated by perikinetic mechanism; When $P e>>1$, it can be assumed that orthokinetic mechanism governs the coagulation process; When $P e \sim 1$, both mechanisms are equally important. Melis et al. ${ }^{15}$ theoretically analyzed the effect of hydrodynamics on the coagulation of two equal-sized particles subject to interaction forces of DLVO type by means of numerically solving the Eq. (1), and proposed a modified expression for the Peclet number

$$
P e^{\prime}=P e \frac{1+\kappa r}{(\kappa r)^{2}}
$$

where $\kappa$ is the inverse double layer thickness and $r=\frac{r_{i}+r_{j}}{2}$. The modified expression shows that the ionic strength (account for the inter-particle forces) of the dispersion medium plays a role in determing the importance of fluid motion in addition to the temperature, medium viscosity, particle diameter and shear rate. Melis et al. ${ }^{15}$ suggested $P e^{\prime}>0.1$ as an approximate criterion for the coagulation rate to be dependent on fluid motion, and the condition $P e^{\prime}<0.1$ allows to exclude all those operating conditions where fluid dynamics affects particle coagulation. 
All these works based on the assumption of perikinetic coagulation employed Fuchs-Smoluchowski's coagulation model. The difference among them was how the inter-particle interactions were accounted for. The majority emplolyed DLVO theory to account for the inter-particle interactions, nevertheless, some others preferred Non-DLVO based methods ${ }^{4,24,25}$. One needs to be careful when applying this assumption as the aforementioned modelling works have applied only to small vessels. Pohn et al ${ }^{41}$ showed that as reactors are scaled up using a popular criterion, i.e. constant power per unit volume, the flow regime can change from laminar in small vessels, to transitional or even turbulent flow in larger ones. If not properly accounted for, this change in flow regime could provoke an evolution of the way the particles eventually coagulate in larger scale vessels. Researchers were divergent in modelling orthokinetic coagulation. Elgebrandt et al. ${ }^{49}$, Krutzer et al. ${ }^{45}$ and Matějíček et al. ${ }^{44}$ adopted Smoluchowski’s shear-induced coagulation model, and Chung et al. ${ }^{3}$ and Lattuada et al. ${ }^{50}$ introduced adjustable parameter collision efficiency into this model in order to better accord with experimental results, while some others $43,46,47,51$ attempted to use semi-empirical coagulation models. The particle coagulation as a result of combined mechanisms received far less attention up to date as can be noted in Table 1 . Initially, semi-empirical coagulation modelling ${ }^{43,51}$ was attempted when studying systems where both mechanisms are thought to be relevant. Vaccaro et al. ${ }^{52}$ assumed the two mechanisms were additive, Forcolin et al. ${ }^{35}$, Lattuada et al. ${ }^{50}$ and Ariafar ${ }^{53}$ employed combined kernels derived from full numerical simulations. It is important to note that many physico-chemical parameters are used in the mathematical modelling of colloidal coagulation. Usually some of them were obtained from direct experimental measurements, while some others were esimtated a priori or by fitting experimental data. Note that care must be taken when validating models that combine particle nucleation, growth and coagulation. Fortuny et al. ${ }^{29,40}$ showed that the dynamics of particle creation and coagulation are such that it is extremely difficult to "see" the real number of particles in the reactor at a given instant, so simply curve fitting complex models to particle size data can 
lead to incorrect parameter estimation. Therefore, it has been suggested that each particular phenomenon should be validated independently ${ }^{29}$.

Different particle coagulation mechanisms are described by different mathematical models, model developments for perikinetic mechanism, orthokinetic mechanism and combined mechanism are thus discussed separately in what follows.

\subsection{Perikinetic coagulation model}

Smoluchowski ${ }^{8}$ developed a perikinetic model as

$$
\beta\left(r_{i}, r_{j}\right)_{\text {peri }}=\frac{2 k_{B} T}{3 \mu}\left(r_{i}+r_{j}\right)\left(\frac{1}{r_{i}}+\frac{1}{r_{j}}\right)
$$

In this equation, inter-particle interactions and particle-particle hydrodynamics were neglected. Several underlying assumptions should be mentioned: the fluid flow is laminar, the collision efficiency is unity, collision occurs between two spherical particles and particles remain spherical after collision.

Muller ${ }^{56}$ and Fuchs ${ }^{9}$ later extended Eq. (5) to include stability ratio as

$$
\beta\left(r_{i}, r_{j}\right)_{\text {peri }}=\frac{2 k_{B} T}{3 \mu W_{i j}}\left(r_{i}+r_{j}\right)\left(\frac{1}{r_{i}}+\frac{1}{r_{j}}\right)
$$

where stability ratio $\left(W_{i j}\right)$ is the inverse of the collision efficiency and accounts for the effects of colloidal and hydrodynamic interactions.

Modelling works incorporating perikinetic coagulation of emulsion polymerizations are based on Eq. (6) $4,23-31,36-40,57-60$. The differences among them are the methods to compute the stability ratio $W_{i j}$ for the specific systems involved. The authors essentially treated $W_{i j}$ as an adjustable 
parameter for the different electrostatic, steric and electrosteric stabilization systems operated in batch, semibatch and continuous reactors, and were thus able to model the behavior of the polymerizations of interest.

It is widely accepted that $W_{i j}$ can be estimated by considering colloidal interactions as follows 27-29, 32, 35-37, 39, 40, 54, 59, 61-63.

$$
W_{i j}=\left(r_{i}+r_{j}\right) \int_{r_{i}+r_{j}}^{\infty} \frac{e^{\frac{V_{\text {int }}}{k_{B} T}}}{R^{2}} d R
$$

While some studies $23,26,30,31,50,60,64$ estimated $W_{i j}$ using the simplified form as:

$$
W_{i j}=\frac{r_{i}+r_{i}}{4 \kappa r_{i} r_{j}} e^{\frac{V_{\mathrm{int,max}}}{k_{B} T}}
$$

Some other works ${ }^{4,25}$ chose a simple empirical approach to estimate $W_{i j}$ as:

$$
W_{i j}= \begin{cases}W_{i j, u u} & \left(r_{i}, r_{j}^{\prime}<r^{*}\right) \\ W_{i j, u s} & \left(r_{i}<r^{*} \text { or } r_{j}^{\prime}>r^{*}\right) \\ \infty & (\text { otherwise })\end{cases}
$$

where $r^{*}$ was critical particle size defined, $r_{i}, r_{j}^{\prime}<r^{*}$ was defined as unstable populations, $r_{j}^{\prime}>r^{*}$ was stable populations, $W_{i j, \text { uu }}$ is the stability ratio between two unstable particles and $W_{i j, u s}$ is the stability ratio between one unstable particle and one stable particle. The values of $r^{*}$, $W_{i j, u u}$ and $W_{i j, u s}$ were determined by fitting the model to experimental data.

Unzueta and Forcada ${ }^{24}$ related $W_{i j}$ to the emulsifier coverage $(\chi)$ of particle surface by

$$
W_{i j}=K_{g}^{\prime} e^{K_{g}^{\prime \prime} \chi^{0.5}}
$$

where $K_{g}^{\prime}$ and $K_{g}^{\prime \prime}$ were adjustable parameters. 
The application of Eq. (7) or (8) has almost become a standard practice among researchers to compute $W_{i j}$, in which the key step is the estimation of total inter-particle interactions $\left(V_{\text {int }}\right)$. The total potential energy of interaction is the net sum of all attractive and repulsive contributions, including van der Waals attraction and electrostatic, steric, depletion and solvation-based repulsion $^{22}$.

The DLVO theory ${ }^{10}$ was usually used to account for van der Waals attractive potential energy $\left(V_{\mathrm{A}}\right)$ and repulsive potential energy $\left(V_{\mathrm{R}}\right)$ due to electrostatic charges on the particle surface in ionic surfactant stabilized latexes. In cases where non-ionic surfactant is used alone, in combination with an ionic surfactant, or if the stabilization is assured by polymer molecules adsorbed on the particle surfaces, steric potential energy $\left(V_{\mathrm{S}}\right)$ should be accounted for. The electrostatic stabilization is far more effective for a given concentration at low to moderate solid contents than the steric mechanism. Neverthless, steric stabilization has some advantages over the electrostatic stabilization as well. For example, one of the most important advantages is that steric stabilization is equally effective in both aqueous and non-aqueous dispersion media, and is relatively insensitive to electrolyte concentrations ${ }^{65,66}$. It also does not exhibit long range interactions between neighbouring particles, and therefore often provides greater stabilization at high solid contents than the electrostatic mechanism does. Some studies included depletion potential energy $\left(V_{\mathrm{D}}\right)$ arising from the presence of free polymer in the solution as well ${ }^{67}$, as it was found that the existence of such free polymers at low concentrations can increase or decrease stability to the latexes ${ }^{68-70}$.

Though DLVO theory is widely used, one must be clearly aware of its strengths and shortcomings 5,71 . DLVO theory does not provide accurate predictions of the aggregation rates of colloids when the potential barrier is located at surface distances below $1 \sim 2 \mathrm{~nm}$ and non-DLVO forces 
dependent on the structure of the solution and particle surface become important. For instance, Pailthorpe and Russel ${ }^{72}$ computed the dispersion forces for polystyrene spheres in water using spectral absorption data. They observed significant retardation effects for $l>5 \mathrm{~nm}(l$ is distance away from particle surface) after putting their results into the DLVO framework, and also found the Hamaker "constant" was a function of the average separation distance and polymer considered. Behrens et al. ${ }^{73}$ argued that DLVO theory overestimated the sensitivity to variation in ionic strength, and the effect of particle size on the double layer repulsion has not been experimentally confirmed. They ${ }^{73,74}$ closely examined the influence of $\mathrm{pH}$ and ionic strength on the stability ratio of carboxylated polystyrene, and polystyrene particles respectively, and concluded that the DLVO theory only worked for low ionic strength and low surface charge conditions. The DLVO theory breaks down beyond these conditions due to the fact that the potential barrier was located at surface distances below $1 \sim 2 \mathrm{~nm}$, where non-DLVO forces existed ${ }^{75}$. In addition, DLVO theory assumes dilute dispersions in which the influence of surrounding particles on a pair of interacting particles can be neglected. In concentrated systems (e.g. high solid content latexes), the surrounding particles can cause an effective reduction in $V_{\text {int }}$, leading to a lower stability ratio ${ }^{76,77}$. This may lead to an overestimation of rate of coagulation. Furthermore, in the application of DLVO theory, it is usually assumed that every particle in the system has the same surfactant coverage, while in reality the precursor particles formed from homogeneous nucleation may undergo coagulation at a rate faster than surfactant diffusion ${ }^{78,79}$.

Non-DLVO effects such as solvation, hydration or structural forces that are significant only at small distances from a particle surface are usually ignored in stability calculation, while these forces might have profound influences on the stability in some situations such as dealing with large polymers or highly hydrophilic polymers ${ }^{80}$. For example, the force between macroscopic surfaces in cyclohexane at separation below $5 \mathrm{~nm}$ was found to oscillate in magnitude and sign 
with distance ${ }^{81}$. Israelachvili and Pashley ${ }^{82}$ found similar oscillations in $10^{-3} \mathrm{M} \mathrm{KCl}$ solution at $\mathrm{pH}=5.5$ when the separation is below $1.5 \mathrm{~nm}$. For a complete overview of the failings of DLVO theory, as applicable to emulsion polymerization, refer to Vale and McKenna's review of PBEs in emulsion polymerization ${ }^{5}$.

Despite this, DLVO theory remains the only basis for non-empirical modeling available for stability ratio and it is possible to correctly predict experimental results by treating certain model constants as adjustable parameters.

\subsection{Orthokinetic model}

Based on the same assumptions involved in Eq. (5), Smoluchowski ${ }^{8}$ proposed an orthokineic model for laminar shear flows

$$
\beta\left(r_{i}, r_{j}\right)_{\text {ortho }}=\frac{4}{3}\left|\frac{\partial U_{x}}{\partial y}\right|\left(r_{i}+r_{j}\right)^{3}
$$

Only one component of the relative velocity was considered in Eq. (11). Hu and Mei ${ }^{83}$ found that the results of Eq. (11) were inaccurate for monodisperse systems because of the inclusion of self-collision ${ }^{84,85}$. The generalized orthokinetic model was given by Camp and Stein ${ }^{86}$ :

$$
\beta\left(r_{i}, r_{j}\right)_{\text {ortho }}=\frac{4}{3} G\left(r_{i}+r_{j}\right)^{3}
$$

Matejicek et al. ${ }^{44}$, Krutzer et al. ${ }^{45}$ and Mayer et al. ${ }^{48}$ used Eq. (12) in their dynamic simulations of limited particle coagulation in emulsion polymerizations. Chung et al. ${ }^{3}$ introduced the parameter collision efficiency $(\alpha)$ into Eq. (12) to simulate the ABS latex coagulation process:

$$
\beta\left(r_{i}, r_{j}\right)_{\text {ortho }}=\frac{4}{3} \alpha G\left(r_{i}+r_{j}\right)^{3}
$$

Chung et al. ${ }^{3}$ employed a spatially averaged shear rate: 


$$
G=\sqrt{\frac{P}{V \mu}}
$$

where $P$ is the agitation power, $V$ the fluid volume and $\mu$ the fluid dynamic viscosity. Note that Eq. (14) is valid for laminar, turbulent and transitional flows ${ }^{87}$. While, the drawback of this approach is the parameter of collision efficiency needs to be estimated based on the experimental data. Note that in industrial scale reactors, especially those used for commodity products that are on the order of several cubic meters, or larger, in volume, using spatially averaged parameters to describe flow-related quantities can be dangerous as the flow fields in such reactors is rarely uniform.

\subsubsection{Laminar flow coagulation model}

In early stages, Camp and Stein ${ }^{86}$ calculated $G$ for laminar flows as:

$$
\Phi=\mu G^{2}=\mu\left[\left(\frac{\partial U_{x}}{\partial y}+\frac{\partial U_{y}}{\partial x}\right)^{2}+\left(\frac{\partial U_{x}}{\partial z}+\frac{\partial U_{z}}{\partial x}\right)^{2}+\left(\frac{\partial U_{y}}{\partial z}+\frac{\partial U_{z}}{\partial y}\right)^{2}\right]
$$

where $\Phi$ is viscous energy dissipation rate per unit volume for laminar flows. However, Eq. (15) was found to be inaccurate in many studies ${ }^{88-92}$. Pedocchi and Piedra-Cueva ${ }^{88}$ proved the validity of Eq. (12) to laminar flow conditions provided $\Phi$ was calculated as:

$$
\Phi=\mu\left[\begin{array}{l}
2\left(\frac{\partial U_{x}}{\partial x}\right)^{2}+2\left(\frac{\partial U_{y}}{\partial y}\right)^{2}+2\left(\frac{\partial U_{z}}{\partial z}\right)^{2}+\left(\frac{\partial U_{x}}{\partial y}+\frac{\partial U_{y}}{\partial x}\right)^{2} \\
+\left(\frac{\partial U_{x}}{\partial z}+\frac{\partial U_{z}}{\partial x}\right)^{2}+\left(\frac{\partial U_{y}}{\partial z}+\frac{\partial U_{z}}{\partial y}\right)^{2}
\end{array}\right]
$$

\subsubsection{Turbulent flow coagulation model}

Eq. (12) can also be applied to turbulent flows, where $G$ can be calculated as ${ }^{43}$

$$
G=\sqrt{\frac{2 \varepsilon}{15 v}}
$$


where $\varepsilon$ is the energy dissipation rate in turbulent flows and $v$ the kinematic viscosity. As far as turbulent flows are concerned, Eq. (12) is only valid for the cases when the particle size is smaller than the Kolmogorov microscale ${ }^{93}$. Some other restrictions should be borne in mind when applying Eq. (12) to turbulent flows; namely that particles must be neutrally buoyant and spherical, there are no large spatial variations in the energy dissipation, and the flow is characterized by high Reynolds numbers leading to isotropic turbulence ${ }^{89}$. As the latex particles are usually small in emulsion polymerizations, the flow around particles was often assumed to be extensional flow field in establishing the coagulation model in turbulent conditions because the extensional flow well reproduces the structure of turbulent flow below the scale of the smallest eddies ${ }^{94}$, which is the scale relevant for the motion of the latex particles ${ }^{15}$.

Saffman and Turner ${ }^{95}$ and Spielman ${ }^{96}$ analyzed the particle coagulation in undisturbed turbulent flow field by assuming that the particles smaller than the smallest eddies move like in a shear flow environment, they derived a similar model equation:

$$
\beta\left(r_{i}, r_{j}\right)_{\text {ortho }}=\sqrt{\frac{8 \pi}{15}}\left(\frac{\varepsilon}{v}\right)^{0.5}\left(r_{i}+r_{j}\right)^{3}
$$

Eq. (18) was also based on the assumption of homogeneous isotropic turbulence. Clark ${ }^{89}$ pointed out that for inhomogeneous turbulence, Eq. (18) may underestimate the collision rate.

Argaman and Kaufman ${ }^{97}$ characterized the random motion of particles in turbulent flows by using a coefficient of turbulent diffusion, which resulted in a model for turbulent coagulation:

$$
\beta\left(r_{i}, r_{j}\right)_{\text {ortho }}=4 \pi K_{s} K_{p} r_{k}^{3} G
$$

where $K_{s}$ is a parameter mainly determined by turbulent frequency, $K_{p}$ is a constant and $r_{k}$ is the aggregate size. 
Delichatsios and Probsein ${ }^{98}$ proposed a model for turbulent coagulation based on the binary collision mean free path concepts

$$
\beta\left(r_{i}, r_{j}\right)_{\text {ortho }}=2 \pi r_{i} r_{j} w_{x}^{\prime}
$$

where $w_{x}^{\prime}=\left(r_{i}+r_{j}\right) \sqrt{\frac{\varepsilon}{15 v}}$ for particles smaller than the smallest eddies.

Kramer and Clark ${ }^{91}$ showed that the collision frequency of particles is a function of the strain rates imposed on the fluid volume element when the fluid is exposed to linear velocity-gradients. A scalar maximum principle strain rate $\left|a_{\max }^{\prime}\right|$ was used to accurately estimate the total collision rate:

$$
\beta\left(r_{i}, r_{j}\right)_{\text {ortho }}=\frac{4 \pi}{3}\left|a_{\max }^{\prime}\right|\left(r_{i}+r_{j}\right)^{3}
$$

Mei and $\mathrm{Hu}{ }^{99}$ supported the results of Eq. (12) and Eq. (18) by employing a numerical approach for uniform, laminar shear flow and Gaussian isotropic turbulence, respectively. They derived a formulation for sheared homogenous turbulence:

$$
\beta\left(r_{i}, r_{j}\right)_{\text {ortho }}=\left[1.2944^{2.2}+\left(\frac{1.333 G}{(\varepsilon / v)^{0.5}}\right)\right]^{1 / 2.2}\left(r_{i}+r_{j}\right)^{3}(\varepsilon / v)^{0.5}
$$

It is worth pointing out that this formulation is applicable to both isotropic and anisotropic turbulences, which introduces an error of approximately $10 \%$ due to the averaging of the transitional effect from isotropic to anisotropic turbulence.

As the latex particles (nano-scale) usually are smaller than the smallest eddies in emulsion polymerizations, only the relevant orthokinetic models those fit this assumption are discussed above. One can see that the inter-particle interactions and particle-particle hydrodynamics were 
not taken into account in Eq. (11) Eq. (22), so the stability ratio needs to be considered when applying these models to latexes. To the best of our knowledge, the orthokinetic model in transitional flows has not been attempted in the literature.

\subsubsection{Empirical coagulation model}

Note that the solid content was not taken into account in the previously mentioned models. Lowry et al. 43,51 investigated the agitation-induced coagulation in stirred vessels. They assumed the perikinetic coagulation was insignificant, and developed a shear-induced coagulation model as:

$$
\beta\left(r_{i}, r_{j}\right)_{\text {ortho }}=\frac{4 G f(\phi)}{\pi W_{i j}}
$$

where $f(\phi)=\phi$ was used ( $\phi$ is solid volume fraction). Chern et al. ${ }^{46,47}$ applied this model to simulate the shear-induced coagulation process in seeded semibatch emulsion polymerizations of acrylic monomers. They found that Eq. (23) failed to predict the coagulum experimental data at higher solid contents (> 40\%), so they proposed a new $f(\phi)$ in order to match model predictions with the experimental data at higher solid contents $(>40 \%)$ :

$$
f(\phi)=\exp \left(\alpha \frac{U_{s}(t=0)}{U_{s}(t)}\right)
$$

where $U_{s}=2\left[\left(74 \rho_{s} / w\right)^{1 / 3}-1\right], w=\frac{\phi \rho_{s}}{\phi \rho_{s}+(1-\phi) \rho_{m}}, \quad \rho_{s}$ is density of solid particle, $\rho_{m}$ is density of the medium, $U_{\mathrm{s}}$ can be considered as a relatively mean free path length between particles.

Empirical models can provide good accord with experiments though, they are invariably based on bench scale measurements and often are system-specific, which may make their extrapolated applications to industrial-scale reactors and different chemical systems be risky. Therefore, due 
caution should be exercised in applying the emipical coagulation models to large industrial-scale reactors and systems other than the orginal ones the models were based on.

\subsubsection{Differential sedimentation coagulation model}

The inertial and gravitational forces of latex nano-particles often can be reasonably neglected. However, centrifugal separation of latex particles is a common industrial technique, in which the centrifugal force is equivalent to imposing a gravitational force. It might need careful attention where its effect can be significant. Possible particle collision and coagulation can be resulted from different terminal velocities in such processes, so the differential sedimentation model might be useful to tackle this effect ${ }^{86,95,100}$. It is noted that there was no modelling work on coagulation of emulsion polymers using the differential sedimentation coagulation model in the open literature. The discussions about the preferential concentration model for weakly inertia particles and accelerative-corrected model for high inertia particles can be referred to Meyer and Deglon 101.

Relatively a number of orthokinetic coagulation models have been proposed (Eq. (11) Eq. (24)) in the literature, neverthess, only very few studies 3,43-49,51 modeled the coagulation using orthokinetic mechanism (by neglecting perikinetic mechanism) in the context of emulsion polymerizations. One possible reason is that the emulsion polymerization applications rarely operate in the regimes governed by orthokinetic mechanism $(P e>>1)$. While, more often than not the situations encountered can reasonably be assumed that the perikinetic coagulation is the dominant mechanism $(P e<<1)$ as it is reflected by the number of studies using perikinetic coagulation model in Table 1. Even though reasonable simplifications may be made, one needs to bear in mind that Brownian motion and fluid motion are always simultaneously present in any practical reactors, and there are cases in which all the effects are nonnegligibly important. 


\subsection{Perikinetic-orthokinetic combined model}

The perikinetic and orthokinetic mechanisms are asymptotic limiting cases where the particle Peclet number is either zero or infinity. The two mechanisms usually codetermine the evolution of PSD in actual coagulation processes especially under industrially relevant operating conditions (e.g., large reactors where local shear rate differs significantly). However, whenever shear forces are present, the evaluation of particle stability is much more difficult, and even simple criteria are not available in the literature ${ }^{102}$. Therefore, many efforts were devoted to the studies of combined coagulation model. Initially, the contributions from the two mechanisms were assumed to be independent and directly additive ${ }^{103}$ :

$$
\beta\left(r_{i}, r_{j}\right)_{\mathrm{com}}=\beta\left(r_{i}, r_{j}\right)_{\text {peri }}+\beta\left(r_{i}, r_{j}\right)_{\text {ortho }}
$$

which can be rewriten as

$$
\beta\left(r_{i}, r_{j}\right)_{\mathrm{com}}=\beta\left(r_{i}, r_{j}\right)_{\mathrm{peri}}\left(1+K_{\mathrm{o}}^{\prime} P e\right)
$$

where $K_{\mathrm{o}}^{\prime}$ is a numerical constant depending on the type of fluid motion. It is a convenient representation to use, and was thus adopted in some studies ${ }^{52,104-106}$. However, van de Ven and Mason ${ }^{107-109}$ performed perturbation analysis of the Fuchs-Smoluchowski convection-diffusion equation in the range of very small Peclet number $(P e \ll<1)$ and they found that shear enhanced the coagulation rate and the enhancement was proportional to the square root of the Peclet number:

$$
\beta\left(r_{i}, r_{j}\right)_{\mathrm{com}}=\beta\left(r_{i}, r_{j}\right)_{\text {peri }}\left(1+K_{\mathrm{o}}^{\prime \prime} P e^{0.5}\right)
$$

where $K_{\mathrm{o}}^{\prime \prime} \quad$ is a numerical constant depending on the type of fluid motion. They concluded that the perikinetic and orthokinetic mechanisms were not directly additive for systems in which Brownian diffusion is the dominant mechanism $(P e<<1)$. Zinchenko and Davis ${ }^{13,14}$ derived a similar equation as Eq. (27) for coagualation rate at small Peclet number conditions $(P e<<1)$. 
Feke and Schowalter ${ }^{54}$ calculated the effect of a small amount of Brownian diffusion on shear-induced coagulation of spherical particles in the regime of large Peclet number $(P e \gg 1)$ based on the perturbation method, they demonstrated that the Brownian contribution is not linearly independent from the shear-induced contribution:

$$
\beta\left(r_{i}, r_{j}\right)_{\mathrm{com}}=\beta\left(r_{i}, r_{j}\right)_{\text {ortho }}\left(1+K_{\mathrm{p}}^{\mathrm{\prime \prime}} P e^{-1}\right)
$$

where $K_{\mathrm{p}}^{\prime \prime}$ is a numerical constant depending on the type of fluid motion. Their work showed that Brownian diffusion can act to increase or to decrease coagulation rate as the flow number is increased. Feke and Schowalter ${ }^{55}$ later performed experiments with monodisperse system to test the two-term perturbation theory, and found the experimental data matched the predictions for $P e$ $\geqq 60$ (shear rate greater than $600 \mathrm{~s}^{-1}$ ). At lower values of shear rate Eq. (28) was not believed to be applicable. Zinchenko and Davis ${ }^{13,14}$ proposed a refined version for $P e>>1$ :

$$
\beta\left(r_{i}, r_{j}\right)_{\text {com }}=\beta\left(r_{i}, r_{j}\right)_{\text {ortho }}\left(1+K_{1}^{\prime \prime} P e^{-1 / 2}+K_{2}^{\prime \prime} P e^{-2 / 3}\right)
$$

where $K_{1}^{\prime \prime}$ and $K_{2}^{\prime \prime}$ are adjustable parameters that depend on the flow condition.

Sheng-Hua et al. ${ }^{110}$ examined the applicability of the combined kernels developed by van de Ven and Mason ${ }^{107-109,111}$ and Zinchenko and Davis ${ }^{13,14}$ for small and large Peclet numbers, and found that these two models can successfully describe the real coagulation feature, but only for the early stages of coagulation.

Zinchenko and Davis ${ }^{13,14}$ numerically simulated the collision rates of small spherical drops and bubbles, and their results further supported the two mechanisms are closely coupled in the regime of finite Peclet values $(0.1 \leq P e \leq 70)$. They proposed a correlation model to approximate the combined coagulation rate for the regime of finite Peclet values $(0.1 \leq P e \leq 70)$ based on the 
detailed numerical simualtions:

$$
\beta\left(r_{i}, r_{j}\right)_{\text {com }}=\beta\left(r_{i}, r_{j}\right)_{\text {ortho }}+\beta\left(r_{i}, r_{j}\right)_{\text {peri }}\left(1+K_{\mathrm{o}}^{\text {" }} P e^{0.5}\right)
$$

Pnueli et al. ${ }^{112}$ extended the theory of coagulation between particles of unequal size by taking into account the modification in the flow field imposed by the larger particle and the effect of Brownian diffusion of the small particle on the coagulation rate, and a coagulation model representing the combined effect was developed:

$$
\beta\left(r_{i}, r_{j}\right)_{\mathrm{com}}=\pi r_{i}^{3} \sqrt{\frac{\varepsilon}{3 v}}\left[4.74 P e^{-2 / 3}+7.5\left(\frac{r_{j}}{r_{i}}\right)^{2}\right]
$$

Melis et al. ${ }^{15}$ and Baldyga et al. ${ }^{16}$ studied the variation of coagulation rate as a result of the changes in Peclet number numerically, and developed a semi-empirical expression by including the perikinetic and orthokinetic effects:

$$
B\left(r_{i}, r_{j}\right)_{\mathrm{com}}=\frac{2 k_{B} T}{3 \mu W_{i j}} \frac{\left(r_{i}+r_{j}\right)^{2}}{r_{i} r_{j}}+a \text { e }^{8.86}
$$

where $\& \&=\frac{7}{6 \sqrt{15}} S \sqrt{\frac{\varepsilon}{v}}$ is the deformation rate, $S$ is a skewness factor for the distribution of $\&$, usually set equal to 0.6 and $a$ is a parameter. It is noted that the exponent of $\not \&(0.86)$ coincides with the one obtained by Zeichner and Schowalter ${ }^{113}$ through a trajectory analysis. Melis et al. ${ }^{15}$ found that Eq. (30) worked properly and matched the numerical simulation results of the convection diffusion equation for unstable latex, while deviated from the numerical simulations to a large extent for more stable systems. This quantitatively illustrated that convective and diffusive mechanisms interact with each for slowly aggregating systems and the additive assumption was not valid for such systems. Based on the model developed by Melis et al. ${ }^{15}$, Forcolin et al. ${ }^{35}$ simulated the particle coagulation phenomenon in asymmetrical extensional flow, and they concluded that the agreement with experimental data was only qualitative. 
Zaccone et al. ${ }^{102,114}$ attempted to formulate the analytical expression for the rate of coagulation in the presence of both shear and Brownian effect based on the simplified form of the convection diffusion equation as:

$$
\frac{\beta\left(r_{i}, r_{j}\right)_{\mathrm{com}}}{\beta\left(r_{i}, r_{j}\right)_{\text {fast }}}=\frac{1}{2 \int_{2}^{2+\delta} \frac{\int_{2+\delta}^{\xi}\left(-P e \cdot f(\xi)+\frac{d\left(\frac{V_{\text {int }}}{k_{B} T}\right)}{d \xi}\right) d \xi}{H(\xi, \lambda) \xi^{2}} d \xi}
$$

where $\delta=\infty$ when $f(\xi)=-\frac{1}{\xi^{2}}$, while $\delta=a \sqrt{\frac{2}{\operatorname{Pe\kappa }\left(r_{i}+r_{j}\right)}}(a$ is a pre-factor, assumed to be $a \approx 1)$ when $f(\xi)=-\frac{\xi(1-A(\xi, \lambda))}{H(\xi, \lambda) \xi^{2}}, A(\xi, \lambda)$ and $H(\xi, \lambda)$ are hydrodynamic interactions, $\xi=\frac{2 R}{r_{i}+r_{j}}, \lambda=\frac{r_{i}}{r_{j}}, R$ is center-to-center separation, $B\left(r_{i}, r_{j}\right)_{\text {fast }}=\frac{2 k_{B} T}{3 \mu} \frac{\left(r_{i}+r_{j}\right)^{2}}{r_{i} r_{j}}$.

This model accounts for the interplay between hydrodynamics and DLVO interactions in a relatively rigorous manner, however it is often not accurate in the high Peclet number regime, i.e., $P e>100^{12}$.

If the complex velocity function in Eq. (33) is replaced by a symmetrical velocity gradient, the following simplified expression can be obtained:

$$
\frac{\beta\left(r_{i}, r_{j}\right)_{\mathrm{com}}}{\beta\left(r_{i}, r_{j}\right)_{\mathrm{fast}}}=\frac{1}{2 \int_{2}^{\infty} \frac{e^{\left(\frac{V_{\text {int }}}{k_{B} T}-\frac{8 P e}{3 \pi \xi}\right.}}{H(\xi, \lambda) \xi^{2}} d \xi}
$$

Later, the same group Lattuada and Morbidelli ${ }^{12}$ solved the Fuchs-Smoluchowski convection-diffusion equation numerically, and derived an expression for the coagulation rate: 


$$
\frac{B\left(r_{i}, r_{j}\right)_{\text {com }}}{B\left(r_{i}, r_{j}\right)_{\text {fast }}}=\frac{\exp \left(H e ( 2 + \delta - \xi ) \left(\int _ { 2 + \delta } ^ { \xi } \left(-P e \cdot f(\xi)+\frac{\left.\left.\left.d \frac{V_{\text {int }}}{d \xi}\right) d \xi-P e \frac{\alpha}{2+\delta}\right)-P e \cdot H e(\xi-2-\delta) \frac{\alpha}{\xi}\right)}{H(\xi, \lambda) \xi^{2}} d \xi\right.\right.\right.}{\left.2 \int_{2}^{\infty} \frac{1}{H\left(k^{2}\right.}\right)}
$$

where $\mathrm{He}$ is the Heaviside function (which is equal to 0 for $x<0$ and equal to 1 for $x>0$ ). The model can, in principle, be used over the entire range of $P e$ values. Two parameters ( $\alpha$ and $a$ ) appear in Eq. (35). $\alpha$ is proportional to the collision efficiency of the coagulation process in the convection controlled regime, and $a$ is an adjustable parameter used in calculating the thickness of the boundary layer $(\delta)$. The two parameters are adjustable, and their values for different types of flows can be referred to Lattuada and Morbidelli ${ }^{12}$. Eq. (35) is applicable to a broad range of conditions. Their effort is one of the most advanced numerical studies of flow-induced coagulation in the presence of perikinetic mechanism to date ${ }^{115}$.

Though some attempts were made to formulate combined models (Eq. (25) - Eq. (35)), their applications to emulsion polymerizations are still rather scarce (see Table 1). Based on the assumption that the total rate of coagulation in shear-induced environment was result of linear summation of perikinetic and orthokinetic mechanisms, Lowry et al. ${ }^{51}$ developed a semi-theoretical equation to better describe the experimental coagulation rate, which showed acceleration in the later stages of coagulation. Vaccaro et al. ${ }^{52}$ adopted Eq. (25) in their theoretical analysis of coagulation phenomenon in mixing controlled operations. Forcolin et al. ${ }^{35}$ incorporated Eq. (32) into a kinetic model for the emulsion polymerization of vinyl chloride monomer. Their model was shown to be able to account for the main different mechanism regulating the coagulation at the same time, i.e., Brownian diffusion, fluid motion and inter-particle interactions, which represented a significant improvement over the pure perikinetic model and orthokinetic model. Recently, Ariafar ${ }^{53}$ tested Eq. (34) in CFD simulations for 
prediction of latex particle coagulation by means of coupling flow field with population balance model in a bench scale stirred reactor. Unfortunately, her simulations were not validated by comparing to experimental data.

\subsection{CFD-based approach}

The vast majority of modeling attempts in Table 1 employed dynamic models, which are based on the assumption of perfect mixing within the reactor. However it should be pointed out that such an assumption is only approximately valid for well-stirred, small-scale reactors. It is most likely invalid for pilot and industrial scale applications. The problem was that the scale-effect of the reactor was not accounted for, and parameter fittings for satisfactory predictions were frequently applied. In addition, only spatially averaged shear rates over the reactor volume were used in the relevant coagulation modelling works (e.g., ${ }^{3,48,51}$ ), nevertheless, the shear rates and energy dissipation rates actually vary spatially in the reactor (see Figure 1).

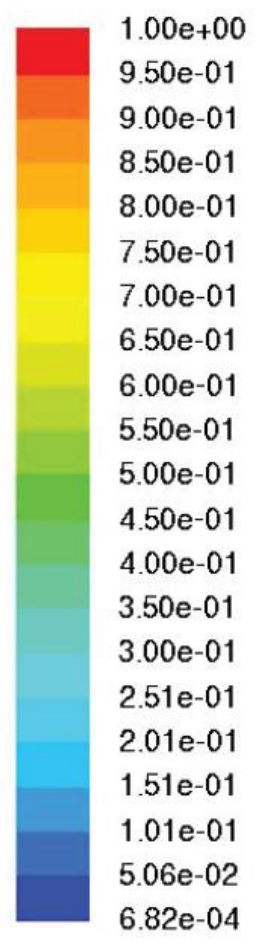

(a). turbulent energy dissipation rate
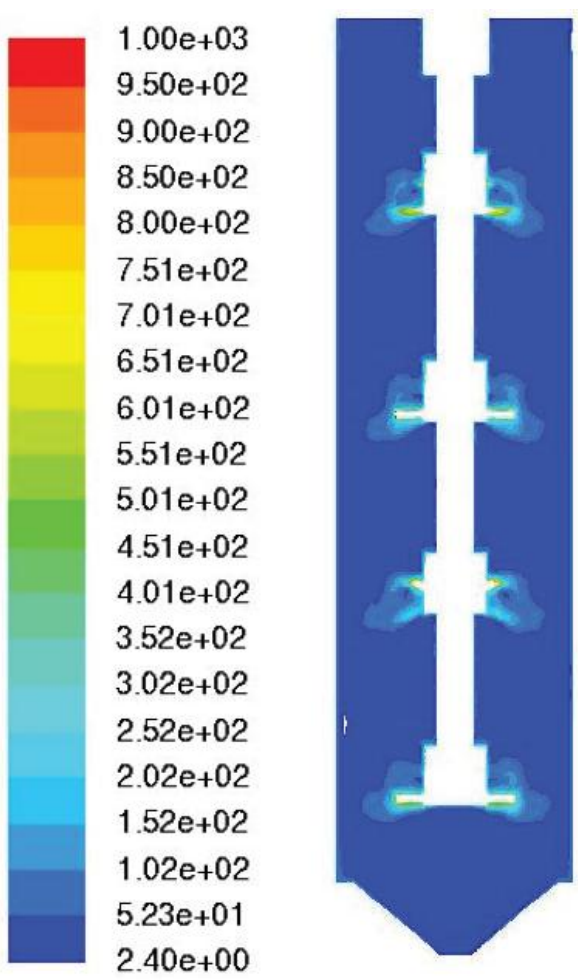

$2.40 \mathrm{e}+00$

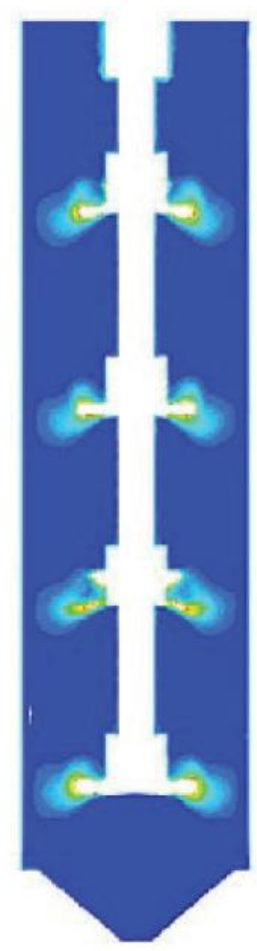


scale reactor ${ }^{53}$.

It can be seen from Figure 1, that even in a bench scale reactor $(D=10 \mathrm{~cm}, H=50 \mathrm{~cm})$, the energy dissipation rates and shear rates near impellers are thousands times those of regions away from impellers. In practice, inhomogeneous flow, species concentration and temperate field prevail in the reactor, which do not only play a significant role in mass and heat transfer, but also govern the dynamic viscosity of the dispersions due to the non-Newtonian rheology ${ }^{116-118}$. This is especially pertinent for high solid content systems in the inherently transient process of emulsion polymerizations. Moreover, the measurement of the viscosities and local shear rates is practically challenging for industrial reactors. It is not difficult to see that all these points underline the limited suitability of the averaged shear rate approach in the prediction of coagulation in emulsion polymerization reactors.

With the rapid advancement of computer performance, computational fluid dynamics (CFD) has become a powerful workhorse to analyze detailed flow field and mixing characteristics within chemical reactors. The appealing aspects of CFD approach are that the effects of material property, geometry, operation condition and reactor scale can be well accounted for by numerically solving the fundamental transport equations. The wide use of the CFD approach has brought about better prediction reliability, more confident scale-up, improved process performance and higher production productivity for chemical industries ${ }^{119,120}$. Coupling the kinetics with the detailed flow field within CFD framework is perceived as a new approach to obtain a deeper insight into the process of emulsion polymerizations. CFD based modeling inherently takes into account the scale-effect of the reactors, and could be applied to industrial vessels. Several attempts were made to model particle coagulation in emulsion polymerization processes based on the CFD approach. 


\subsubsection{Multizonal one way coupling approach}

Elgebrandt et al. ${ }^{49}$ developed a hybrid-multizonal framework enabling solving the detailed kinetic model based on the obtained flow field from commercial CFD package FLUENT. The shear induced coagulation model (Eq. (21)) was used and the Dougherty-Krieger equation ${ }^{121}$ for hard spheres was used to estimate the viscosity of the dispersion. They found that, as one would expect, higher shear rates caused increased coagulation.

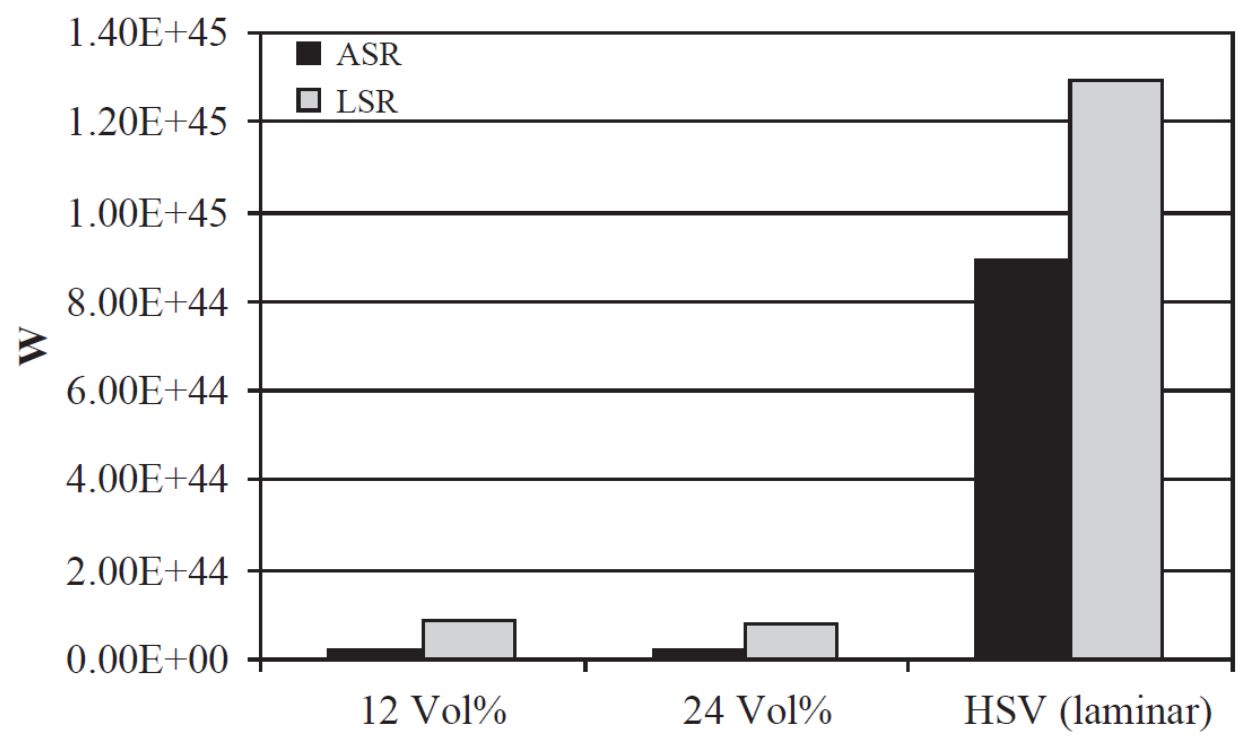

Figure 2. Comparison of the stability ratio obtained from CFD simulations by using the ASR and the LSR methods at $N=400 \mathrm{rpm}^{49}$.

The stability ratios were calculated using both the ASR (average shear rate) and the LSR (local shear rate) methods as shown in Figure 2 (HSV stands for high solid volume fraction). As seen, the stability ratio is under-predicted by around $45 \%$ by using the ASR method, while the under-prediction was about $380 \%$ and $350 \%$ for the 12 vol. $\%$ and 24 vol. \% case, respectively.

Alexopoulos et al. ${ }^{33}$ developed a two-compartment nonhomogeneous mixing model to calculate the dynamic evolution of PSD in an industrial scale semibatch emulsion copolymerization of 
BuA/MMA reactor. The multi-compartment model parameters were extracted from CFD simulations of the flow field in the emulsion polymerization reactor as developed earlier in Alexopoulos et al. ${ }^{122}$, and the emulsion polymerization reaction kinetics were coupled with particle nucleation, growth and coagulation. Perikinetic coagulation model (Eq. (6)) was used. The spatial variations of PSD in the large scale reactor due to non-ideal mixing were accounted for by the multi-compartment model.

Pohn et al. ${ }^{41}$ developed a multi-zonal population balance model (PBM) for an emulsion polymerization stirred reactor, which imported flow field from the commercial CFD package. The non-Newtonian rheology for high solid content system was modeled by the Carreau-Yasuda model. Nucleation and growth was neglected, only particle coagulation (Eq. (6)) was taken into account in the PBM model, in which inter-particle interaction forces were described by the DLVO theory. They investigated the particle coagulation behavior induced by adding salt solution into the vessel operated in laminar flow regime and examined the effect of reactor scale on the final PSD of the high solid content latexes. A typical result is shown in Figure 3.

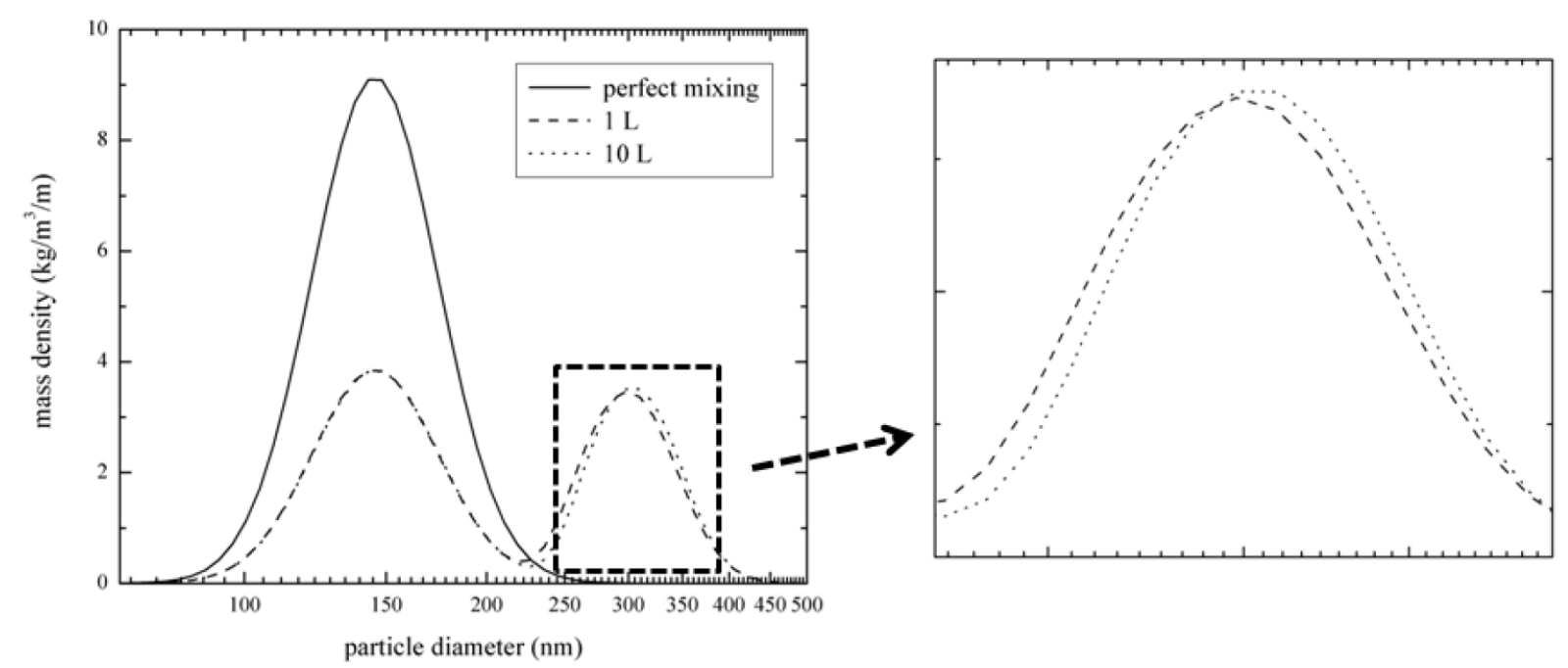

Figure 3. Comparison of PSDs at the same time $(t=215 \mathrm{~s})$ in three vessels using the same simulation conditions ${ }^{41}$. 
The result of a perfectly mixed tank was compared to those obtained from $1 \mathrm{~L}$ and $10 \mathrm{~L}$ vessel, where the time-dependent dynamics of mixing can't be ignored. The comparison showed that mixing conditions and reactor scale can have a big impact on the PSD obtained during a coagulation process. The same authors later incorporated nucleation and growth kinetics into the multi-zonal population balance model ${ }^{123,124}$, and further studied the effect of reactor scale and inhomogeneous mixing on the PSD of the latex dispersions. However, their model was not validated by comparing to experimental measurements.

\subsubsection{Two way coupling approach}

In order to investigate the particle coagulation phenomena in a more detailed manner, Ariafar ${ }^{53}$ developed a two-way coupling approach connecting flow field CFD simulation with population balance model in the presence of only particle coagulation term. The perikinetic kernel (Eq. (6)), orthokinetic kernel (Eq. (18)) and combined kernel (Eq. (34)) were tested, the impacts of reactor scale and imperfect mixing conditions on the evolution of the latex PSD were explored.
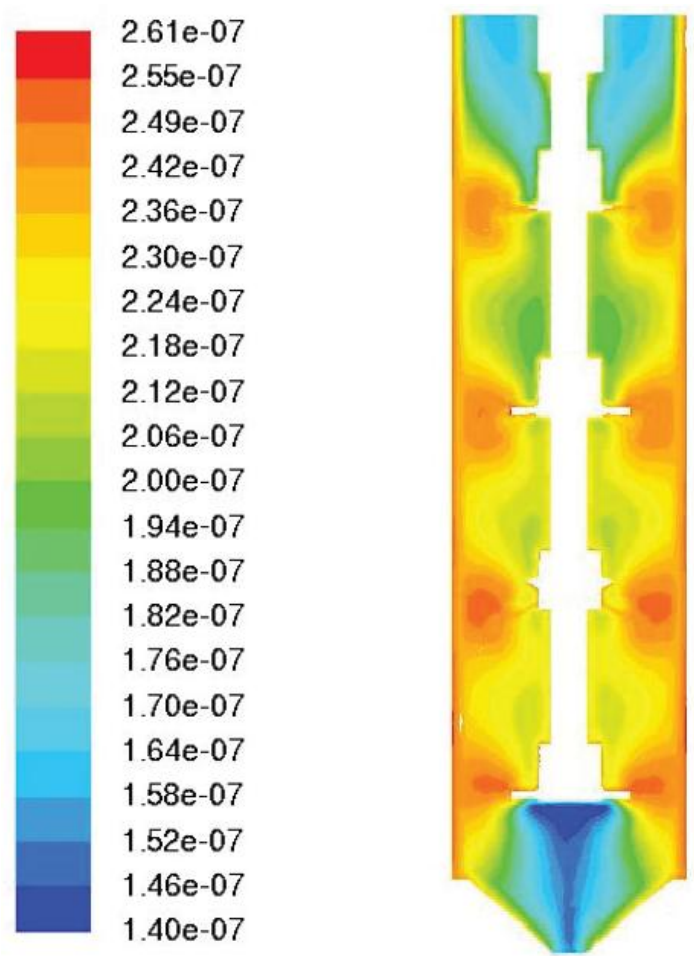

Figure 4. Contour of mean particle diameter at $t=5$ s predicted using Eq. (18) ${ }^{53}$. 
Figure 4 shows the distribution of mean particle diameter after $5 \mathrm{~s}$ of mixing a pre-defined latex under conditions of shear induced coagulation (Eq. (18)). The initial condition was set as mean diameter of $65 \mathrm{~nm}$ with normal distribution everywhere in the domain. As can be seen, the particles are bigger in the regions where energy dissipation rates are larger. Their simulations were constrained to use short time frames and excluded nucleation and particle growth due to such two-way coupling simulations are exceedingly heavy.

\subsection{Brownian dynamics approach}

The Brownian dynamics simulation is a mesoscopic method in which the dispersion medium molecules are replaced instead by a stochastic force. That means the Brownian motion of the colloidal particles produced by random collisions with dispersion medium molecules is mimicked by a stochastic force generated by pseudo-random numbers. In this way, the Brownian dynamics approach can describe the dynamic behavior of individual particles whose mass and size are larger than those of the dispersion medium molecules. This method takes advantage of the fact that there is a large separation in time scales between the rapid motion of medium molecules and the more sluggish motion of colloidal particles. As a general rule, particles are assumed to be in thermal equilibrium with the dispersion medium and is subject to an external force $\mathbf{F}$. The translational motion of a particle is thus described by the Langevin equation as

$$
\frac{d\left(m_{p} u_{k}\right)}{d t}=\mathbf{F}_{k}-\varsigma m_{p} u_{k}+X_{k}
$$

where LHS is the inertial force of particle of mass $m_{p}$, the first term on the RHS is systematic external force, the second term on the RHS is the frictional drag of the fluid around the particle, $\varsigma$ is the friction constant and the last term is a random acceleration force caused by the random 
collisions between particle and the dispersion medium molecules.

The Brownian dynamics simulations of aggregation kinetics of thousands of particles were attempted $^{125-128}$, in which the independent trajectories of particles were tracked by solving the Langevin stochastic differential equation and collision among particles were explicitly taken into account at discrete time intervals. The pairwise potential interaction, hydrodynamic interaction and diffusion effect can be explicitly modeled in such methods. Bos et al. ${ }^{125}$ and Puertas et al. ${ }^{126}$ carried out Brownian dynamics simulations for thousands of particles to describe the first stages of cluster formation, and hence understand their structures at different volume fractions and interaction potentials. Puertas et al. ${ }^{127}$ and Romero-Cano et al. ${ }^{80}$ employed Brownian dynamics simulations to examine the influence of steric interactions in the initial stages of aggregation kinetics in two-particle systems. Liu and Larson ${ }^{129}$ studied the recognition kinetics between two patterned colloidal spheres using Brownian dynamics simulation with full hydrodynamic interactions. Mohammadi et al. ${ }^{115}$ investigated the binding kinetics in the dilute solid content limit by considering interactions of two spheres under shear flow across the entire range of Peclet numbers. However, the particle rotational motion and contact effect such as adhesive and elastic forces was not considered in the Brownian dynamics simulations. Furthermore, such method is limited to systems with small inertia.

\subsection{Discrete element method}

The discrete element method (DEM) is a numerical method for computing the motion and effect of a large number of small particles. Compared with Brownian dynamics simulations, the method is generally distinguished by its inclusion of rotational degrees-of-freedom as well as stateful 
contact and often complicated geometries. The advantage of this methid is the direct incorporation of the contact, non-contact inter-particle forces and torques into the model, which enables it directly accounts for the particle-based phenomena such as crowding and stabilization of the particles without simpilications that are often used ${ }^{114,130,131}$. The particle trajectories are governed by the well-known Newton's equation of motion

$$
\frac{d^{2} \mathbf{x}_{i}}{d t^{2}}=\frac{\mathbf{F}_{i}}{m_{i}}
$$

where $\mathbf{F}_{i}$ represents the total forces acting on the discrete particle $i$. The balance of angular momentum $\left(\mathbf{L}_{i}\right)$ for each particle can be written as

$$
\frac{d \mathbf{\Omega}_{i}}{d t}=\frac{\mathbf{M}_{i}}{I_{i}}
$$

where $\boldsymbol{\Omega}_{i}=\mathbf{L}_{i} / I_{i}$ is the rotation rate of particle $i, \mathbf{M}_{i}$ is the total torques acting on particle $i$, and $I_{i}$ is the particle momentum of inertia.

Recently, discrete element method (DEM) was used to model the colloidal systems in the context of emulsion polymerizations (e.g., Figure 5). Kroupa et al. ${ }^{132}$ simulated the particle coagulation phenomenon of concentrated colloidal dispersions employing the DEM method. Interaction potentials were described by DLVO theory and the contact forces were accounted for by using JKR theory ${ }^{131,133}$. 


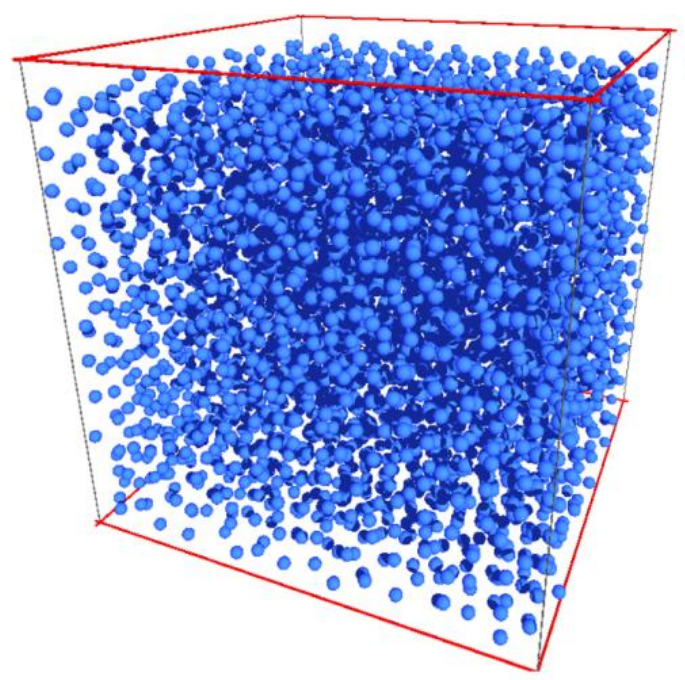

(a) $t=0 \mathrm{~s}$

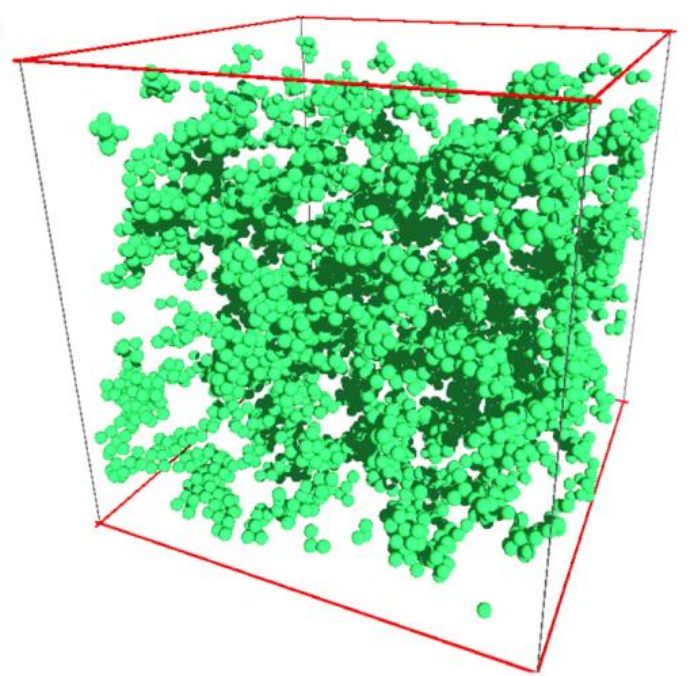

(b) $t=900 \mu \mathrm{s}$

Figure 5. Snapshots from DEM simulation with $N_{\mathrm{p}}=5000$ primary particles under a shear rate of

$$
8 \times 10^{4} \mathrm{~s}^{-1} 134
$$

It was shown that the coagulation behaviour was strongly dependent on the solid content, the surface potential and the shear rate. They demonstrated that the doublet formation rate was insufficient for the description of the coagulation kinetics of latexes. In addition, the detailed DEM model was shown to be able to explain the autocatalytic nature of the coagulation of stabilized latexes subjected to shear. They later ${ }^{134}$ modeled the coagulation behaviour of a dispersion with moderate particle volume fraction subjected to high shear rates using DEM, their results revealed that the coagulation was not only affected by shear rate, but also by the degree of particle adhesion. The rheological behaviour of concentrated dispersions under high shear was investigated as well by means of DEM simulation ${ }^{135}$. The viscosity was found to be independent of shear rate and primary particle size for monodisperse system, while the viscosity grew rapidly with solid content. The results illustrate that the correct description of the coagulation kinetics requires the consideration of the whole system with its complexity. So the DEM method is a great tool for this purpose, which is completely based on the physics and employs no fittings parameters ${ }^{132}$. However, because of the lack of the experimental data in the field of the complex 
fluid rheology, the thorough validation of the DEM predictions is yet to be done. Besides, the particle nucleation, deformation and growth are challenging to be taken into account in the framework of DEM simulation. The disadvantage of this approach is the necessity of computing with a very small time step $\left(\Delta t \approx 1 \times 10^{-10} \mathrm{~s}\right)$, which imposes high demand for computational resources ${ }^{132,134,135}$. Worse still, it becomes even more computationally expensive as the number of particles increases.

\section{Experimental studies}

All variables including the concentration and type of monomer, surfactant, buffer, initiator, the type of reactor, and the operating conditions (temperature, stirring rate, pressure, flow rates, ...) etc. can influence coagulation phenomenon and hence the final PSD of emulsion polymerizations. A slight change of one of these factors may lead to a tremendous variation in the final PSD of the latex products. Many experimental studies, therefore, were carried out to obtain quantitative information and insights into the coagulation processes of the emulsion polymerizations and correlating different variables to evolutions of PSD.

\subsection{Ab initio/seeded emulsion polymerizations}

The relevant experimental studies concerned with the particle coagulation phenomena in the emulsion polymerizations during the past decades are summarized in Table 2. One can note that the particle coagulation was either coupled with both nucleation and growth sub-processes (i.e., ab initio emulsion polymerization) or only coupled with growth sub-process (i.e., seeded

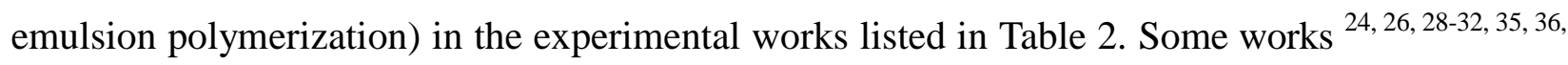
136-141 discussed the evolution of PSD and total number of particles with emulsion polymerizations under the influence of particle coagulation, and the correlation between coagulation phenomena and various recipe/operating conditions. While, some others $43,44,46,47$, 
142-144 particularly studied the formation of coagulum casued by particle coagulation in emulsion polymerizations and correlated its formation with operating conditions, especially the agitation intensity.

Table 2. Experimental studies on PSD evolution of emulsion polymerizations with emphasis laid on particle coagulation (N, G, C signify nucleation, growth and coagulation, respetively).

\begin{tabular}{|c|c|c|c|c|c|}
\hline Refs & Reactor & Monomers & Surfactant & Initiator & Mechanisms \\
\hline 142 & Semibatch & $\begin{array}{l}\mathrm{BuA} / \mathrm{AA} \\
\mathrm{BuA} / \mathrm{SEMA}\end{array}$ & Anionic & APS & $\mathrm{N}, \mathrm{G}, \mathrm{C}$ \\
\hline 43 & Batch & $\mathrm{VC} / \mathrm{EA}$ & Non-ionic & APS & $\mathrm{N}, \mathrm{G}, \mathrm{C}$ \\
\hline 44 & Semibatch & Sty/BuA/AA & Anionic & APS & $\mathrm{N}, \mathrm{G}, \mathrm{C}$ \\
\hline 46,47 & Semibatch & MMA/BuA & Anionic & $\mathrm{NaPS}$ & $\mathrm{N}, \mathrm{G}, \mathrm{C}$ \\
\hline 24 & Semibatch & $\begin{array}{l}\text { MMA/ } \\
\text { BuA }\end{array}$ & $\begin{array}{l}\text { Anionic/ } \\
\text { Non-ionic }\end{array}$ & APS & $\mathrm{N}, \mathrm{G}, \mathrm{C}$ \\
\hline 136,137 & Batch & Sty/VAc & Anionic & $\mathrm{NaPS}$ & $\mathrm{G}, \mathrm{C}$ \\
\hline 30,31 & Batch & VAc & $\begin{array}{l}\text { Anionic/ } \\
\text { Non-ionic }\end{array}$ & APS & $\mathrm{N}, \mathrm{G}, \mathrm{C}$ \\
\hline 36 & Semibatch & $\mathrm{VAc} / \mathrm{BuA}$ & Non-ionic & APS & $\mathrm{N}, \mathrm{G}, \mathrm{C}$ \\
\hline 35 & Batch & $\mathrm{VC}$ & Anionic & APS & $\mathrm{N}, \mathrm{G}, \mathrm{C}$ \\
\hline 138 & Semibatch & $\mathrm{BuA}$ & Anionic & $\begin{array}{l}\mathrm{KPS} / \\
\mathrm{SBc}\end{array}$ & $\mathrm{G}, \mathrm{C}$ \\
\hline 143 & Loop Reactor & $\mathrm{VAc} / \mathrm{BuA}$ & $\begin{array}{l}\text { Anionic/ } \\
\text { Non-ionic }\end{array}$ & KPS & $\mathrm{N}, \mathrm{G}, \mathrm{C}$ \\
\hline 143 & Semibatch & Sty/BuA & Anionic & KPS & $\mathrm{N}, \mathrm{G}, \mathrm{C}$ \\
\hline 144 & Semibatch & Sty/BuA & $\begin{array}{l}\text { Anionic/ } \\
\text { Non-ionic }\end{array}$ & $\begin{array}{l}\text { APS/ } \\
\text { SM }\end{array}$ & $\mathrm{G}, \mathrm{C}$ \\
\hline 26 & Semibatch & Sty & Anionic & APS & $\mathrm{N}, \mathrm{G}, \mathrm{C}$ \\
\hline 28 & Batch & Sty/EA & Non-ionic & APS & $\mathrm{N}, \mathrm{G}, \mathrm{C}$ \\
\hline 29 & Batch & BuA/MMA & $\begin{array}{l}\text { Anionic/ } \\
\text { Non-ionic }\end{array}$ & APS & $\mathrm{N}, \mathrm{G}, \mathrm{C}$ \\
\hline 139 & Batch & $\mathrm{VC}$ & Anionic & KPS & $\mathrm{N}, \mathrm{G}, \mathrm{C}$ \\
\hline 32 & Batch & Sty & $\begin{array}{l}\text { Anionic/ } \\
\text { Non-ionic }\end{array}$ & KPS & $\mathrm{N}, \mathrm{G}, \mathrm{C}$ \\
\hline 140 & Batch & $\begin{array}{l}\text { AcA/ } \\
\text { DMAEA }\end{array}$ & Non-ionic & APS & $\mathrm{N}, \mathrm{G}, \mathrm{C}$ \\
\hline 141 & Batch & Sty & Anionic & $\begin{array}{l}\text { KPS/ } \\
\text { AIBN }\end{array}$ & $\mathrm{N}, \mathrm{G}, \mathrm{C}$ \\
\hline
\end{tabular}

The knowledge obtained from these efforts is undoubtedly useful for product development and improved operations of relevant emulsion polymerization productions. Since a plethora of 
sub-processes are involved in emulsion polymerization processes, the complex interplay among all these sub-processes determines the final PSD of the latexes. Though a considerable amount of knowledge was obtained, a problem in interpreting the results obtained from these studies is that the particle coagulation is coupled with particle growth and/or nucleation sub-processes, it is difficult to identify the exact contribution of particle coagulation. Therefore, works were carried out to examine the coagulation phenomenon independently.

Pure perikinetic coagulation was independently studied by considering non-reacting nanoparticle latexes in quiescent medium (e.g., in a very small cell or tube). Hidalgo-Alvarez et al. ${ }^{145}$ extensively reviewed on the effects of various physicochemical factors on the perikinetic coagulation of colloidal latexes ${ }^{146,147}$. A majority of those works dealt with coagulation in dilute conditions, and studied the effects of surfactant type, particle surface properties, the type of surface group, surface charge sign/value, free molecules, and temperature etc. on coagulation kinetics and behavior. Only a limited number of papers worked on concentrated solid volume fraction systems ${ }^{61,148,149}$.

Since the perikinetic and orthokinetic mechanisms are always present simultaneously in any practical process such as emulsion polymerization, the relevant works are discussed next.

\subsection{Experimental studies on salt induced coagulation}

Salt-induced coagulation discussed here was resulted from perikinetic and orthokinetic combined mechanisms where both mechanisms are non-negligible. Particle coagulation in emulsion polymerization reactors in such intermediate range was less focused on. The coagulation was studied by means of adding (one step or stepwise) a known amount of electrolyte solution into the stabilized latex in the reactor. Samples were taken at fixed time intervals in order to monitor the 
evolution of PSD and hence the coagulation behavior. Such experimental efforts are listed in Table 3.

Table 3. Experimental studies on salt induced coagulation in emulsion polymerization reactors

(N, G, C signify nucleation, growth and coagulation, respetively).

\begin{tabular}{|l|l|l|l|l|l|}
\hline Refs & Reactor & System & Surfactant & Mechanisms & Electrolyte \\
\hline 136 & Batch & $\begin{array}{l}\text { Sty/ } \\
\text { VAc }\end{array}$ & Anionic & C & Pre-charged Salt \\
\hline 137 & Batch & $\begin{array}{l}\text { Sty/ } \\
\text { VAc }\end{array}$ & Anionic & C & Pre-charged Salt \\
\hline 37 & Batch & VAc & Anionic & C & Add Salt \\
\hline 150 & Batch & Sty/AA & Anionic & C & Pre-charged Salt \\
\hline 40 & Batch & BuA/MMA & $\begin{array}{l}\text { Anionic/ } \\
\text { Non-ionic }\end{array}$ & C & Add Salt \\
\hline 151 & Batch & Sty/AA & Anionic & C & Pre-charged Salt \\
\hline 52 & Semibatch & VDC & Anionic & C & Add Salt \\
\hline 152 & $\begin{array}{l}\text { Batch/ } \\
\text { CSTR }\end{array}$ & Sty & - & C & Add Salt \\
\hline 153 & Semibatch & VDF & - & C & Add Salt \\
\hline
\end{tabular}

Reynhout et al. ${ }^{150}$ studied the strength of the electrostatic repulsion and steric repulsion forces as a function of the electrolyte concentration, $\mathrm{pH}$, and the temperature for polystyrene (PS) and styrene/acrylic acid copolymer (PS/AA) latexes. They concluded that the low-pH PS/AA latex remained stable against coagulation at high electrolyte concentration due to the presence of steric repulsion. The low-pH PS/AA latex lost its stability when temperature was higher than the critical coagulation temperature $\left(35^{\circ} \mathrm{C}\right)$. While, the high-pH PS/AA latex was still stable when the temperature went up to $85{ }^{\circ} \mathrm{C}$ and the concentration of electrolyte was high, which was attributed to the strong electro-steric stabilization. The same authors ${ }^{151}$ later demonstrated that the presence of surface-bound carboxylic groups only improved the colloidal stability under the conditions that the carboxylic groups were charged (i.e., at a high $\mathrm{pH}$ ), while the PS/AA latex was less stable than the PS latex at a low pH. Kemmere et al. ${ }^{136,137}$ studied the coagulation behavior of 
polystyrene and polyvinyl acetate latexes at relatively low and higher solid contents by performing batch seeded swelling experiments, they found no dependency of the coagulation behavior on process conditions and actually electrolyte concentration dominated the coagulation processes. Melis et al. ${ }^{37}$ analyzed the coagulation behavior by stepwise adding a known amount of electrolyte solution into the latexes stabilized by anionic surfactant, they showed that the enhancement of the coagulation rate by shear is negligible under low Peclet number conditions. Using a similar experimental approach, Fortuny et al. ${ }^{40}$ investigated the effects of three stabilizing species including sulfate macro-radicals, SDS (electrostatic), and TA (electro-steric) surfactants on the stability of BuA-MMA copolymer latexes. They reported that the stability provided by the sulfate end groups was negligible compared to the contributions of either of the anionic surfactants, and the highest stability was obtained from TA surfactant. Vaccaro et al. ${ }^{52}$ examined the aggregation behavior of PVDC dispersion of monodispersed systems induced by the salt solution (aluminum nitrate nonahydrate). When the salt solution and stable latex were pumped into the reactor simultaneously, they observed that the steady-state aggregate size was insensitive to the stirring speed at high solid volume fractions in the feed, while it became quite sensitive to stirring speed at low solid volume fraction. It was found that particles coagulated instantaneously at high feeding solid volume fractions, and particles coagulated completely before mixing at small scales was achieved by feeding stabilized latex into the reactor pre-filled with salt solution above the critical coagulation concentration (CCC). Soos et al. ${ }^{152}$ studied aggregation behavior of aggregates produced from fully destabilized polystyrene latex particles in turbulent flows. They showed that the collision efficiency depended on the shear rate $\left(\alpha \propto G^{-0.18}\right)$, and the steady-state values of two measured moments of the cluster mass distribution (CMD) were fully reversible upon a change in stirring speed. The results illustrated that the aggregate structure was independent of the shear rate in the given range of stirring speeds although the moments of CMD at steady-state depended on the appied shear rate. Méndez-Ecoscia et al. ${ }^{153}$ 
recently proposed a new methodology to evaluate the in-situ variation of latex stability by means of interpretating the backscattering profiles obtained using a Turbiscan Lab ${ }^{\circledR}$ device in a rapid and robust manner. This method can be employed as a rapid and reproducible technique to evaluate important parameters such as CCC, stability ratio and Hamaker constant (A) used in the modelling of particle coagulation phenomenon.

\subsection{Experimental studies on shear coagulation}

Shear-induced (or flow-induced) coagulation discussed here falls into the regime where shear dominated the process. Such relevant studies are summarized in Table 4.

Table 4. Experimental studies on shear-induced coagulation

(N, G, C signify nucleation, growth and coagulation, respetively).

\begin{tabular}{|l|l|l|l|l|l|}
\hline Refs & Reactor & System & Surfactant & Mechanisms & Electrolyte \\
\hline 51 & Batch & VC/EA & Non-ionic & C & Pre-charged Salt \\
\hline 154 & Couette & ABS & Anionic & C & Pre-charged Salt \\
\hline 155 & Couette & Sty & - & C & Pre-charged Salt \\
\hline 45 & Couette & Sty & Non-ionic & C & Pre-charged Salt \\
\hline 156 & Batch & Sty & - & C & Pre-charged Salt \\
\hline 3 & Batch & ABS & Anionic & C & Pre-charged Salt \\
\hline 157 & Couette & Sty & - & C & Pre-charged Salt \\
\hline 158 & Microchannel & Sty/Ac & Anionic & C & - \\
\hline 159 & Microchannel & Sty/Ac & - & C & - \\
\hline 114 & Couette & Sty/Ac & - & C & Pre-charged Salt \\
\hline 160 & Couette & Sty/Ac & - & C & Pre-charged Salt \\
\hline 161 & Couette & AcA & - & C & Pre-charged Salt \\
\hline 162 & Microchannel & MMA/Sty & Anionic & C & - \\
\hline 163 & Batch & BA/MMA & Anionic & C & Pre-charged Salt \\
\hline 164 & Couette & MMA/BuA & - & C & Pre-charged Salt \\
\hline
\end{tabular}

Lowry et al. ${ }^{51}$ investigated mechanical coagulation of latexes both in the presence and absence of air-liquid interface in stirred tanks. Various electrolyte concentrations were used to adjust the level of stability of the latexes such that they could be stored for 2 weeks without any sign of coagulation while coagulated readily when subjected to agitation. Their experiments confirmed 
that mechanical coagulation was related to both shear and surface coagulation and the rate of coagulation could be reduced by $2500 \%$ by eliminating the liquid-air interface. They explained the increase in particle coagulation due to increased agitation to the reason that the air surface area-to-liquid volume was increased by causing surface to ripple to a greater extent. Under shear-controlled regimes, Spicer et al. ${ }^{156}$ investigated the effect of impeller type on the evolution of the average floc size and structure during flocculation of polystyrene particles in stirred tanks, and found that the evolution of floc size was not significantly influenced by impeller types at constant averaged shear rate (i.e., by changing impeller types while keeping the averaged shear rate as constant). Chung et al. ${ }^{3}$ studied the influence of operating variables on particle size distribution of ABS (Acrylonitrile-butadiene-styrene). They observed little effect of the residence times of the continuous processes on particle coagulation under the operating conditions where shear dominated the process dynamics. Urrutia et al. ${ }^{163}$ investigated reactor fouling casused by preformed latexes, they found orthokinetic fouling increased with time, solid content, and temperature, and it decreased as the colloidal stability of the latex increasesd.

The disadvantage of using stirred tank to this effect is that the shear rate is heterogeneously distributed over the tank. Homogenous shear can be generated in Couette reactor (or eventually a rheometer), so this device is used to quantify the single-shear effect on particle coagulation. By using a Couette reactor, Krutzer et al. ${ }^{45}$ found the particle collision was the same for both laminar flow and isotropic turbulent flow, while the shear rate sensed by two particles approaching each other was higher in laminar flow than in turbulent flow at equal energy dissipation. As a consequence, the stability ratio for turbulent flow was lower than that for the laminar flow and the coagulation rate was higher in isotropic turbulent flow. In a latex undergoing rapid shear coagulation, Torres et al. ${ }^{155}$ reported that polystyrene particles with a low surface charge can form strong, rigid bonds and aggregate into large, tenuous flocs. Selomulya et al. ${ }^{157}$ sheared 
latexes composed of particles with diameters of 60,380 and $810 \mathrm{~nm}$, respectively, in a Couette reactor. The aggregates of different primary particle sizes displayed disctinct behavior in attaining steady state under similar shear conditions. The flocs made of smaller particles (60 and $380 \mathrm{~nm})$ were more prone to restructuring instead of fragmentation when compared with those composed of larger particles $(810 \mathrm{~nm})$, particularly at low to moderate shear rates $\left(G \leq 100 \mathrm{~s}^{-1}\right)$. Zaccone et al. ${ }^{114,160}$ observed shear-induced solidification (i.e., explosive increase in viscosity) of dilute charge-stabilized colloids after an induction period during which the viscosity was equal to zero-time value (see Figure 6).

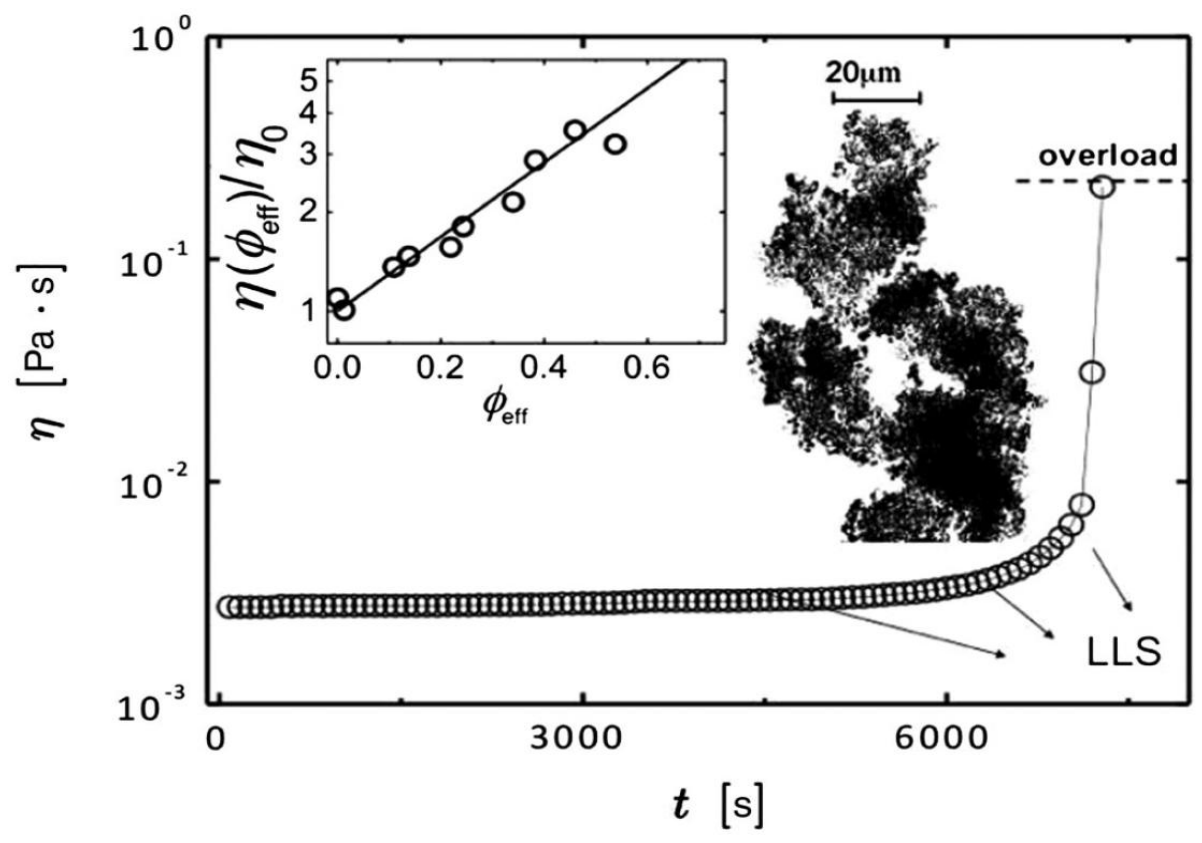

Figure 6. Time evolution of the viscosity at $G=1700 \mathrm{~s}^{-1}$ and $\phi=0.21^{160}$.

The colloidal particles used in this study were surfactant-free polystyrene-acrylate latex spheres stabilized by the charged groups of the initiator. Flow-induced coagulation of dilute colloidal polystyrene nanoparticles dispersed in viscoelastic solutions was explored by Xie et al. ${ }^{161}$. Their results showed that the solution viscosity enhanced the coagulation while elasticity reduced it. Jaquet et al. ${ }^{164}$ recently reported that shear lyophobic colloids can lead to gelation if the formed clusters can grow to sizes large enough to percolate. Ghosh and Lindt ${ }^{154}$ paid particular 
attention to the effect of temperature on the coagulation process of latexes in a Couette reactor, their work has shown that the coagulation temperature controlled the average particle size and set the limit on the particle size distribution and the most effective processing means to control the particle size and the particle morphology was the coagulation temperature.

A Couette apparatus can only provide shear rate as high as several thousands in magnitude. Microchannels were used to generate substantially high shear rate on the order of magnitude of $10^{4} \sim 10^{6} \mathrm{~s}^{-1}$ by forcing the colloidal system to pass through the micro-Homogenizer under high pressure ${ }^{158}$. Zaccone et al. ${ }^{102,114}$ and $\mathrm{Wu}$ et al. ${ }^{158}$ examined the stability behavior in high shear flow generated in microchannel for strongly repulsive systems in dilute and relatively higher solid content conditions without adding any salt, especially the connection between the aggregation/gelation behavior and the surfactant adsorption state on the particle surface. Interesting phenomena were observed when the given colloidal systems with different values of surfactant surface density $(\Gamma)$ sheared through a microchannel at an extremely high shear rate: they become solid-like materials when $\Gamma$ below a certain value, and turn into liquid-like state when $\Gamma$ above this value. Xie et al. ${ }^{159}$ later found this kind of transition (colloidal system to solid-like gel or liquid-like state) was a function of primary particle size and shear rate. High shear induced coagulation was also used to develop new materials. By forcing the different colloidal systems to pass through the micro-Homogenizer under high pressure to generated intense shear $\left(1.5 \times 10^{6} \mathrm{~s}^{-1}\right)$, Meng et al. ${ }^{162}$ successfully prepared composite materials where A nanoparticles are uniformly and randomly distributed inside a matrix of B nanoparticles.

\subsection{Preparation of large monodisperse polymer latexes}

It is well known that emulsion polymerization can be used to prepare small monodispere latexes, with a limited solid content directly. However if one wishes to make larger particles, it is 
necessary to use semibatch or seeded processes, and it becomes more challenging to get monodisperse particles for a number of reasons. ${ }^{165,166}$ At the risk of oversimplifying, a non-monodisperse system comes from different sources: (1) particle initiation that is not instantaneous - i.e. different particles have different ages, and have grown for different times; the longer the initiation period, the broader the PSD; and (2) non-uniform particle coagulation can occur (i.e. some particles coagulate more/less than others, depending on their size). One way to overcome these problems would be to use high temperatures and rapidly decompising initiators. That way particles are nucleated rapidly, and have almost exactly the same size. If particle coagulation occurs, it would be uniform since all of the particles are identical. However, to ensure monodisperse particles, it is usually required to add high amounts of surfactant, thus leading to very small particle sizes ${ }^{166-171}$. In addition one is limited in terms of the upper limit of solid content in such batch systems.

The ionic strength also plays a critical role in determining the final particle size (via latex stability) and needs to be carefully controlled in order to have a very narrow particle size distribution. It is found that the larger monodisperse particles were produced at higher ionic strength conditions, and that the ionic strength needs to be carefully controlled in order to have a very narrow particle size distribution ${ }^{172,182,187}$. By suitable adjustment of the ionic strength of the aqueous phase, the initiator concentration, and the polymerization temperature, it was found possible to obtain a range of particle sizes $(0.1$ to $1.0 \mu \mathrm{m})$ by single-stage reactions ${ }^{172,181,188}$.

In order to prepare particles on the order of $500 \mathrm{~nm}$, the procedure normally used is to prepare a seed dispersion by ab initio emulsion polymerization and then to grow the particles by stages to the required sizes ${ }^{166}$. The seeding technique is recommened to achieve high product uniformity. However, even in seeded experiments, the maximal particles diameter in emulsion polymerization is limited to about $1 \mu \mathrm{m}^{172}$. This is because of creaming and settling of the 
particles, and their sensitivity to mechanic shear ${ }^{166}$.

Surfactant-free proceedures have also been developed with the goal of achieving larger particle sizes $^{173-177}$. In such emulsifier-free methods, the free radicals from the decomposition of the initiator formed end-groups on the polymer chains, and the these end-groups provide the means of rendering the particle colloidally stable ${ }^{173} 178-180$. With the correct formulation, well-defined feed profiles can be used to control the latex coagulation during the polymerization, to prepare monodisperse particles with diameters on the order of $3.0 \mu \mathrm{m}$ in the single-stage soap-free polymerization in aqueous media ${ }^{181-183}$. However, one would expect surfactant-free (or surfactant-poor) systems to have time dependent, complex nucleation steps, and a significant degree of coagulation (which in turn depends on the size of the particles involved in the collisions) 184, 185 . Thus it is not clear that it is particularly attractive in terms of attaining high solid contents and narrow PSD.

\section{Discussions and Perspectives}

The coagulation rate coefficient is the basis for accurate simulation of PSD variation due to particle coagulation in emulsion polymerizations. Experiments are useful in deepening fundamental understanding on this complex phenomenon and hence facilitate mathematical modeling. Despite the advances in the modeling of particle coagulation phenomenon, it still needs further investigation.

The models appearing in the literature to date were derived from the assumption of binary collision of spherical spheres, and remain spherical after collision without taking into consideration of any other realistic factors. The binary collision assumption is only approximately valid for dilute systems, which applies rigorously only in the limit of infinite dilution $\phi \rightarrow 0{ }^{130}$. This is a strong limitation to the applicability of the model to emulsion polymerizations, which 
generally operates at moderate to high solid content (40 vol.\% 60 vol. \%) conditions, especially recent trend is to increase the solid content of latex formulation to as high as $75 \mathrm{vol} . \%$ in order to promote economic productivity ${ }^{123,124}$.

The presence of other particles was shown to affect even binary collisions ${ }^{95}$, and the chances of multiple-particle collisions in moderate and high solid content systems are significantly increased. Different behavior in the kinetics of particle coagulation has been experimentally observed for non-dilute conditions ${ }^{149,189,190}$. It was recognized that the effect of particle concentration on the coagulation phenomenon can't be neglected ${ }^{65}$. Furthermore, the particle shape and deformation can play an important role in the process of coagulation, even the inter-particle interaction potential varies between different geometries ${ }^{191}$, which were not accounted for in the present coagulation models.

The material properties of the medium (e.g., viscosity) interact with the coagulation phenomenon as well ${ }^{49,143,144}$. The apparent viscosity of the reaction mixture increases (especially significantly in high solid systems) during emulsion polymerization, which resulting in a viscous pseudo-plastic reaction mixture ${ }^{192}$. Shear-induced coagulation was reported to be as dramatic as the complete solidification of even dilute systems, which caused explosive increase in viscosity 159, 160, 193 . Meanwhile, the almost fully turbulent flow at the start may turn into much less developed turbulence. The viscoelastic nature of the media was shown to strongly affect the particle coagulation ${ }^{194,195}$. The viscous pseudo-plastic behavior is associated with formation of structural linkages among particles driven by Brownian coagulation ${ }^{196}$, while both shear and Brownian movement can meanwhile in turn damage the linkages between the particles. Some experiments on two-spherical-particle systems showed that attractive interactions existed between particles moving in viscoelastic fluids but not in Newtonian fluids ${ }^{197-199}$. Scirocco et al. ${ }^{200}$ and 
Won et al. ${ }^{201}$ observed particle alignment only in shear-thinning viscoelastic fluids rather than Boger fluids. Xie et al. ${ }^{161}$ investigated the flow-induced coagulation of dilute colloidal polystyrene nanoparticles both in Newtonian and viscoelastic solutions. They found elasticity reduces the particle coagulation while the solution viscosity enhances the coagulation processes. Fundamental understandings on how the viscosity and elasticity influence particle coagulation and verse vice remain unresolved. More quantitative experiments are still needed to justify the interactions between viscosity/elasticity and particle coagulation. This complex interaction between material properties and the particle coagulation was rarely taken into account in modeling of coagulation.

The perikinetic coagulation in dilute stagnant systems is generally thought to have been fairly well understood ${ }^{11}$. However, this is not the case for systems under non-dilute conditions and under shear ${ }^{130}$. Stabilized latexes under shear were found to remain stable for very long time period and then suddenly jam into solid-like pastes ${ }^{114,202,203}$. More fundamental understanding of the coagulation phenomenon under shear, especially in the crowded environment is very much needed, which could in turn shed light on modeling of this phenomenon.

It appears that the peri- and orthokinetic models were more frequently used in emulsion polymerizations (see Table 1). Though there were some attempts to formulate the combined models, but they were still divergent and rarely applied to practical conditions. More works need to be carried out to examine the validity of the combined models by comparing to carefully designed in-house experiments and bring about unified versions, which is of special importance since perikinetic-orthokinetic combined effect may prevail in some practical applications.

Therefore, new coagulation models that can take into account all the realistic physics in such 
practical applications are highly desirable. The application of the present models to practical systems should be with caution, the user needs to be fully aware of the limitations of the models chosen.

On the other hand, it is indeed challenging to solve the mentioned drawbacks and advance the model development theoretically. Semi-empirical correlations can be developed as a step further based on the experimental data obtained from practical systems, but their extrapolation to a wider range of operation conditions can be risky. Fortunately, the emerging DEM simulation has the potential to directly model the particle coagulation phenomenon occurred among a finite number of particles based on the first principle ${ }^{132,134,135}$. It is convenient to artificially control the shear rate and Brownian motion conditions in DEM simulations, so the method can serve as a workhorse to facilitate understanding of the coagulation kinetics and derive new coagulation models in more realistic conditions. The improved coagulation model can be well coupled with CFD framework to simulate the particle coagulation phenomenon with better confidence without using parameter fittings. The process design, improvement and scale up can thus be fairly expected.

\section{Acknowledgements}

We thank ANR for its financial support of project Scale-up (ANR-12-RMNP-0016). 


\section{Nomenclature}

F external force, $\mathrm{N}$

$G \quad$ shear rate, $1 / \mathrm{s}$

$G \quad$ acceleration due to gravity, $\mathrm{m} / \mathrm{s}^{2}$

$H \quad$ hydrodynamic interaction function between two particles

I particle momentum of inertia, $\mathrm{kg} \cdot \mathrm{m}^{2}$

$k_{B} \quad$ Boltzmann constant, $\mathrm{J} / \mathrm{K}$

$K_{g}^{\prime} \quad$ constant

$K_{g}^{\prime \prime} \quad$ constant

$K_{\mathrm{o}}^{\prime \prime}, K_{\mathrm{p}}^{\prime \prime} \quad$ numerical constant depending on the type of fluid motion

$K_{\mathrm{s}}, K_{\mathrm{p}} \quad$ constant

$l \quad$ distance away from particle surface, $\mathrm{m}$

L angular momentum, $\mathrm{kg} \bullet \mathrm{m}^{2} / \mathrm{s}$

$m \quad$ particle mass, $\mathrm{kg}$

M total torques acting on a particle, $\mathrm{N} \bullet \mathrm{m}$

$N \quad$ impeller agitation speed, rpm

$P \quad$ power consumption, $\mathrm{W}$

$P e \quad$ particle Peclet number

$P e^{\prime} \quad$ modified particle Peclet number

$r \quad$ radius of spherical particle, $\mathrm{m}$

$R \quad$ center-to-center separation, $\mathrm{m}$

$t \quad$ time, $\mathrm{s}$

$T \quad$ temperature, $\mathrm{K}$

$u \quad$ particle velocity, $\mathrm{m} / \mathrm{s}$

$U \quad$ velocity component, $\mathrm{m} / \mathrm{s}$

$V \quad$ total volume of liquid, $\mathrm{m}^{3}$

$V_{\mathrm{A}} \quad$ Van der Waals attractive potential energy, $\mathrm{J}$

$V_{\mathrm{D}} \quad$ depletion potential energy, J

$V_{\text {int }} \quad$ total inter-particle interactions, $\mathrm{J}$

$V_{\mathrm{R}} \quad$ repulsive potential energy, $\mathrm{J}$

$V_{\mathrm{S}} \quad$ steric potential energy, $\mathrm{J}$ 


$$
\begin{array}{cc}
W_{i j} & \text { stability ratio } \\
\Delta t & \text { time step, s }
\end{array}
$$

\section{Greek letters}

$\begin{array}{ll}\alpha & \text { collision efficiency } \\ \beta & \text { coagulation kernel } \\ \varepsilon & \text { turbulent kinetic energy dissipation rate, } \mathrm{m}^{2} / \mathrm{s}^{3} \\ \mu & \text { dynamic viscosity, Pa.s } \\ v & \text { kinetic viscosity, } \mathrm{m}^{2} / \mathrm{s} \\ \gamma & \text { deformation rate, } 1 / \mathrm{s} \\ \kappa & \text { inverse double layer thickness, } 1 / \mathrm{m} \\ \phi & \text { solid volume fraction } \\ \varsigma & \text { friction constant } \\ \chi & \text { emulsifier coverage, } \mathrm{mol} / \mathrm{m}^{2} \\ \Phi & \text { viscous energy dissipation } \mathrm{rate} \text { per unit volume for laminar flow } \\ \Gamma & \text { surfactant surface density, } \mathrm{mol} / \mathrm{m}^{2} \\ \rho & \text { density, kg/m } \\ \boldsymbol{\Omega} & \text { rotation rate of particle, } \mathrm{rad} / \mathrm{s}\end{array}$

\section{Subscripts}

$i, j, k \quad$ the $i^{\text {th }}, j^{\text {th }}, k^{\text {th }}$ particle

p particle

$x, y, z \quad$ radial, tangential or axial directions

\section{Abbreviations}

AA acrylic acid

ABS acrylonitrile-butadiene-styrene

Ac acrylate

AcA acrylamide

APS ammonium persulfate 
AIBN azodiisobutyronitrile

BuA butyl acrylate

C coagulation

CFD computational fluid dynamics

CMC critical micelle concentration

DEM discrete element method

DLVO Derjaguin-Landau-Verwey-Overbeek (theory)

DMAEA 2-dimethylamino-ethyl acrylate

EA ethyl acrylate

EHA 2-ethylhexyl acrylate

G growth

KPS potassium persulfate

MMA methyl methacrylate

$\mathrm{N} \quad$ nucleation

NaPS sodium persulfate

PBE population balance equation

PSD particle size distribution

Refs references

$\mathrm{SBc} \quad$ sodium bicarbonate

SEMA sulfoethyl methacrylate

Sty Styrene

SFS sodium formaldehyde sulphoxylate

tBHP t-butyl hydrogen peroxide

VAc vinyl acetate

VC vinyl chloride

VDC vinylidene chloride

VDF vinylidene fluoride

VOC volatile organic compounds 


\section{References}

1. Feedonia: http://www.Freedoniagroup.com/industry-study/world-emulsion-polymers-2929.htm (last accessed October 20, 2017).

2. Blackley, D. C. Polymer Latices: Science and Technology. Vol. 1, Fundamental Principles; Chapman \& Hall: London, 1997.

3. Chung, C. B.; Park, S. H.; Han, I. S.; Seo, Y. H.; Yang, B. T. "Modeling of ABS latex coagulation processes", AIChE J. 1998, 44, 1256-1265.

4. Vale, H. M.; McKenna, T. F. "Particle formation in vinyl chloride emulsion polymerization: Reaction modeling", Ind. Eng. Chem. Res. 2009, 48, 5193-5210.

5. Vale, H.; McKenna, T. "Modeling particle size distribution in emulsion polymerization reactors", Prog. Polym. Sci. 2005, 30, 1019-1048.

6. Ramkrishna, D. Population Balances: Theory and Applications to Particulate Systems in Engineering; Academic Press: New York, 2000.

7. Marchisio, D. L.; Fox, R. O. Multiphase Reacting Flows: Modelling and Simulation; Springer: New York, 2007.

8. Smoluchowski, V. "Grundriß der koagulationskinetik kolloider lösungen", Colloid. Polym. Sci. 1917, 21, 98-104.

9. Fuchs, v. N. " ber die stabilität und aufladung der aerosole", Zeitschrift für Physik A Hadrons and Nuclei 1934, 89, 736-743.

10. Verwey, E. J. W.; Overbeek, J. T. G.; Overbeek, J. T. G. Theory of the Stability of Lyophobic Colloids; Elsevier: New York, 1999.

11. Russell, W. B.; Schowalter, W.; Saville, D. Colloidal Dispersions; Cambridge University Press: Cambridge, 1991.

12. Lattuada, M.; Morbidelli, M. "Effect of repulsive interactions on the rate of doublet formation of colloidal nanoparticles in the presence of convective transport", J. Colloid. Interf. Sci. 2011, 355, 42-53.

13. Zinchenko, A. Z.; Davis, R. H. "Gravity-induced coalescence of drops at arbitrary péclet numbers", J. Fluid Mech. 1994, 280, 119-148.

14. Zinchenko, A. Z.; Davis, R. H. "Collision rates of spherical drops or particles in a shear flow at arbitrary peclet numbers", Phys. Fluids 1995, 7, 2310-2327.

15. Melis, S.; Verduyn, M.; Storti, G.; Morbidelli, M.; Bałdyga, J. "Effect of fluid motion on the aggregation of small particles subject to interaction forces", AIChE J. 1999, 45, 1383-1393.

16. Bałdyga, J.; Jasińska, M.; Krasiński, A.; Rožeń, A. "Effects of fine scale turbulent flow and mixing in agglomerative precipitation", Chem. Eng. Tech. 2004, 27, 315-323.

17. Chern, C. "Emulsion polymerization mechanisms and kinetics", Prog. Polym. Sci. 2006, 31, 443-486.

18. Thickett, S. C.; Gilbert, R. G. "Emulsion polymerization: State of the art in kinetics and mechanisms", Polymer 2007, 48, 6965-6991.

19. Hansen, F.; Ugelstad, J. "Particle nucleation in emulsion polymerization. I. A theory for homogeneous nucleation", J. Polym. Sci. A1. 1978, 16, 1953-1979.

20. Feeney, P. J.; Napper, D. H.; Gilbert, R. G. "Coagulative nucleation and particle size distributions in emulsion polymerization", Macromolecules 1984, 17, 2520-2529.

21. Gilbert, R. G. Emulsion polymerization: A Mechanistic Approach; Academic Press: New York, 1995.

22. Ottewill, R. H. "Stability of polymer colloids", In Polymeric Dispersions: Principles and Applications; Asua, J.M., Eds.; Springer: New York, 1997; pp 31-48.

23. Richards, J. R.; Congalidis, J. P.; Gilbert, R. G. "Mathematical modeling of emulsion copolymerization 
reactors", J. Appl. Polym. Sci. 1989, 37, 2727-2756.

24. Unzueta, E.; Forcada, J. "Modeling the effect of mixed emulsifier systems in emulsion copolymerization", $J$. Appl. Polym. Sci. 1997, 66, 445-458.

25. Araujo, P. H. H.; de la Cal, J. C.; Asua, J. M.; Pinto, J. C. "Modeling particle size distribution (PSD) in emulsion copolymerization reactions in a continuous loop reactor", Macromol. Theor. Simul. 2001, 10, 769-779.

26. Zeaiter, J.; Romagnoli, J.; Barton, G.; Gomes, V.; Hawkett, B.; Gilbert, R. "Operation of semi-batch emulsion polymerisation reactors: Modelling, validation and effect of operating conditions", Chem. Eng. Sci. 2002, 57, 2955-2969.

27. Immanuel, C. D.; Doyle, F. J.; Cordeiro, C. F.; Sundaram, S. S. "Population balance PSD model for emulsion polymerization with steric stabilizers", AIChE J. 2003, 49, 1392-1404.

28. Kammona, O.; Pladis, P.; Frantzikinakis, C. E.; Kiparissides, C. "A comprehensive experimental and theoretical investigation of the styrene/2- ethylhexyl acrylate emulsion copolymerization", Macromol. Chem. Phys. 2003, 204, 983-999.

29. Fortuny, M.; Graillat, C.; McKenna, T. F.; Araújo, P. H.; Pinto, J. C. "Modeling the nucleation stage during batch emulsion polymerization", AIChE J. 2005, 51, 2521-2533.

30. Coen, E. M.; Gilbert, R. G.; Morrison, B. R.; Leube, H.; Peach, S. "Modelling particle size distributions and secondary particle formation in emulsion polymerisation", Polymer 1998, 39, 7099-7112.

31. Coen, E. M.; Peach, S.; Morrison, B. R.; Gilbert, R. G. "First-principles calculation of particle formation in emulsion polymerization: Pseudo-bulk systems", Polymer 2004, 45, 3595-3608.

32. Feiz, S.; Navarchian, A. H. "Emulsion polymerization of styrene: Simulation the effects of mixed ionic and non-ionic surfactant system in the presence of coagulation", Chem. Eng. Sci. 2012, 69, 431-439.

33. Alexopoulos, A. H.; Pladis, P.; Kiparissides, C. "Nonhomogeneous mixing population balance model for the prediction of particle size distribution in large scale emulsion polymerization reactors", Ind. Eng. Chem. Res. 2013, 52, 12285-12296.

34. Pladis, P.; Alexopoulos, A. H.; Kiparissides, C. "Mathematical modeling and simulation of vinylidene fluoride emulsion polymerization", Ind. Eng. Chem. Res. 2014, 53, 7352-7364.

35. Forcolin, S.; Marconi, A.; Ghielmi, A.; Butte, A.; Storti, G.; Morbidelli, M. "Coagulation phenomena in emulsion polymerisation of vinyl chloride", Plast. Rubber. Compos. 1999, 28, 109-115.

36. Lazaridis, N.; Alexopoulos, A.; Chatzi, E.; Kiparissides, C. "Steric stabilization in emulsion polymerization using oligomeric nonionic surfactants", Chem. Eng. Sci. 1999, 54, 3251-3261.

37. Melis, S.; Kemmere, M.; Meuldijk, J.; Storti, G.; Morbidelli, M. "A model for the coagulation of polyvinyl acetate particles in emulsion", Chem. Eng. Sci. 2000, 55, 3101-3111.

38. Immanuel, C. D.; Cordeiro, C. F.; Sundaram, S. S.; Meadows, E. S.; Crowley, T. J.; Doyle, F. J. "Modeling of particle size distribution in emulsion co-polymerization: Comparison with experimental data and parametric sensitivity studies", Comput. Chem. Eng. 2002, 26, 1133-1152.

39. Immanuel, C. D.; Doyle III, F. J. "Computationally efficient solution of population balance models incorporating nucleation, growth and coagulation: Application to emulsion polymerization", Chem. Eng. Sci. 2003, 58, 3681-3698.

40. Fortuny, M.; Graillat, C.; McKenna, T. F. "Coagulation of anionically stabilized polymer particles", Ind. Eng. Chem. Res. 2004, 43, 7210-7219.

41. Pohn, J.; Heniche, M.; Fradette, L.; Cunningham, M.; McKenna, T. "Development of a computational framework to model the scale- up of high- solid- content polymer latex reactors", Chem. Eng. Tech. 2010, 33, 1917-1930. 
42. Aryafar, S.; Sheibat- Othman, N.; McKenna, T. F. "Coupling of CFD simulations and population balance modeling to predict brownian coagulation in an emulsion polymerization reactor", Macromol. React. Eng. 2017, 11, 1600054.

43. Lowry, V.; El- Aasser, M. S.; Vanderhoff, J. W.; Klein, A. "Mechanical coagulation in emulsion polymerizations", J. Appl. Polym. Sci. 1984, 29, 3925-3935.

44. Matějíček, A.; Pivoňková, A.; Kaška, J.; Ditl, P.; Formánek, L. "Influence of agitation on the creation of coagulum during the emulsion polymerization of the system styrene-butylacrylate-acrylic acid", J. Appl. Polym. Sci. 1988, 35, 583-591.

45. Krutzer, L.; Van Diemen, A.; Stein, H. "The influence of the type of flow on the orthokinetic coagulation rate", J. Colloid. Interf. Sci. 1995, 171, 429-438.

46. Chern, C.; Hsu, H.; Lin, F. "Stability of acrylic latices in a semibatch reactor", J. Appl. Polym. Sci. 1996, 60, 1301-1311.

47. Chern, C.; Kuo, Y. "Shear-induced coagulation kinetics of semibatch seeded emulsion polymerization", Chem. Eng. Sci. 1996, 51, 1079-1087.

48. Mayer, M.; Meuldijk, J.; Thoenes, D. "Dynamic modeling of limited particle coagulation in emulsion polymerization", J. Appl. Polym. Sci. 1996, 59, 83-90.

49. Elgebrandt, R. C.; Romagnoli, J. A.; Fletcher, D. F.; Gomes, V. G.; Gilbert, R. G. "Analysis of shear-induced coagulation in an emulsion polymerisation reactor using computational fluid dynamics", Chem. Eng. Sci. 2005, 60, 2005-2015.

50. Lattuada, M.; Zaccone, A.; Wu, H.; Morbidelli, M. "Population-balance description of shear-induced clustering, gelation and suspension viscosity in sheared dlvo colloids", Soft Matter 2016, 12, 5313-5324.

51. Lowry, V.; El-Aasser, M.; Vanderhoff, J.; Klein, A.; Silebi, C. "Kinetics of agitation-induced coagulation of high-solid latexes", J. Colloid. Interf. Sci. 1986, 112, 521-529.

52. Vaccaro, A.; Šefč́́k, J.; Wu, H.; Morbidelli, M.; Bobet, J.; Fringant, C. "Aggregation of concentrated polymer latex in stirred vessels", AIChE J. 2006, 52, 2742-2756.

53. Ariafar, S. Scale-up of Emulsion Polymerization Process: Impact of Changing Characteristic Times; Doctoral Thesis, Univeristy Claude Bernard Lyon 1: Lyon, France, 2016.

54. Feke, D.; Schowalter, W. "The effect of brownian diffusion on shear-induced coagulation of colloidal dispersions", J. Fluid. Mech. 1983, 133, 17-35.

55. Feke, D.; Schowalter, W. "The influence of brownian diffusion on binary flow-induced collision rates in colloidal dispersions", J. Colloid. Interf. Sci. 1985, 106, 203-214.

56. Müller, H. "Zur allgemeinen theorie ser raschen koagulation", Fortschrittsberichte über Kolloide und Polymere 1928, 27, 223-250.

57. Kiparissides, C.; Achilias, D.; Chatzi, E. "Dynamic simulation of primary particle- size distribution in vinyl chloride polymerization", J. Appl. Polym. Sci. 1994, 54, 1423-1438.

58. Kiparissides, C.; Moustakis, I.; Hamielec, A. "Electrostatic and steric stabilization of pvc primary particles", J. Appl. Polym. Sci. 1993, 49, 445-459.

59. Dokucu, M. T.; Park, M.-J.; Doyle, F. J. "Reduced-order methodologies for feedback control of particle size distribution in semi-batch emulsion copolymerization", Chem. Eng. Sci. 2008, 63, 1230-1245.

60. Doyle, F. J.; Harrison, C. A.; Crowley, T. J. "Hybrid model-based approach to batch-to-batch control of particle size distribution in emulsion polymerization", Comput. Chem. Eng. 2003, 27, 1153-1163.

61. Jia, Z.; Gauer, C.; Wu, H.; Morbidelli, M.; Chittofrati, A.; Apostolo, M. "A generalized model for the stability of polymer colloids", J. Colloid. Interf. Sci. 2006, 302, 187-202.

62. Immanuel, C. D.; Doyle, F. J. "A sensitivity approach to reachability analysis for particle size distribution in 
semibatch emulsion polymerization", Ind. Eng. Chem. Res. 2004, 43, 327-339.

63. Overbeek, J. T. G.; Kruyt, H. Colloid Science, Vol. 1: Kinetics of Flocculation; Elsevier: New York, 1952.

64. Ottewill, R. Emulsion Polymerization; Academic Press: New York, 1982.

65. Hirtzel, C.; Rajagopalan, R. "Invited review stability of colloidal dispersions", Chem. Eng. Commun. 1985, 33, 301-324.

66. Zimehl, R.; Lagaly, G. "Coagulation of latex dispersions in the presence of some organic compounds", Colloid. Surface. 1987, 22, 215-224.

67. Seebergh, J. E.; Berg, J. C. "Depletion flocculation of aqueous, electrosterically-stabilized latex dispersions", Langmuir 1994, 10, 454-463.

68. Sato, T.; Ruch, R. Stabilization of Colloidal Dispersions by Polymer Adsorption; Marcel Dekker: New York, 1980.

69. Napper, D. H. Polymeric Stabilization of Colloidal Dispersions; Academic Press: New York, 1983.

70. Poehlein, G. W.; Ottewill, R. H.; Goodwin, J. W. Science and Technology of Polymer Colloids: Characterization, Stabilization and Application Properties; Springer: New York, 2013.

71. Theodoor, J.; Overbeek, G. "Strong and weak points in the interpretation of colloid stability", Adv. Colloid Interf. Sci. 1982, 16, 17-30.

72. Pailthorpe, B.; Russel, W. "The retarded van der waals interaction between spheres", J. Colloid. Interf. Sci. 1982, 89, 563-566.

73. Behrens, S. H.; Christl, D. I.; Emmerzael, R.; Schurtenberger, P.; Borkovec, M. "Charging and aggregation properties of carboxyl latex particles: Experiments versus dlvo theory", Langmuir 2000, 16, 2566-2575.

74. Behrens, S.; Borkovec, M.; Semmler, M. "Aggregation in sulfate latex suspensions: The role of charge for stability predictions", In Trends in Colloid and Interface Science XII; Koper, G. J. M.; Bedeaux, D.; Cavaco, C.; Sager, W. F. C., Eds.; Springer: New York, 1998; pp 66-69.

75. Israelachvili, J. N. Intermolecular and Surface Forces; Academic Press: New York, 2011.

76. Hütter, M. "Coagulation rates in concentrated colloidal suspensions studied by brownian dynamics simulation", Phys. Chem. Chem. Phys. 1999, 1, 4429-4436.

77. Hsu, J.-P.; Liu, B.-T. "Critical coagulation concentration of a colloidal suspension at high particle concentrations", J. Phys. Chem. B 1998, 102, 334-337.

78. Hansen, F.; Ugelstad, J. "Particle nucleation in emulsion polymerization. Iii. Nucleation in systems with anionic emulsifier investigated by seeded and unseeded polymerization", J. Polym. Sci: Polym. Chem. 1979, 17, 3047-3067.

79. Giannetti, E. "Nucleation mechanisms and particle size distributions of polymer colloids", AIChE J. 1993, 39, 1210-1227.

80. Romero-Cano, M.; Puertas, A.; De las Nieves, F. "Colloidal aggregation under steric interactions: Simulation and experiments", J. Chem. Phys. 2000, 112, 8654-8659.

81. Christenson, H.; Horn, R.; Israelachvili, J. "Measurement of forces due to structure in hydrocarbon liquids", J. Colloid. Interf. Sci. 1982, 88, 79-88.

82. Israelachvili, J. N.; Pashley, R. M. "Molecular layering of water at surfaces and origin of repulsive hydration forces", Nature 1983, 306, 249-250.

83. Hu, K. C.; Mei, R. "Particle collision rate in fluid flows", Phys. Fluids 1998, 10, 1028-1030.

84. Sundaram, S.; Collins, L. R. "Collision statistics in an isotropic particle-laden turbulent suspension. Part 1. Direct numerical simulations", J. Fluid. Mech. 1997, 335, 75-109.

85. Collins, L. R.; Sundaram, S. "Comment on "particle collision rate in fluid flows" [Phys. Fluids 10,1028 (1998)]", Phys. Fluids 1998, 10, 3247-3248. 
86. Camp, T. R.; Stein, P. C. "Velocity gradients and internal work in fluid motion", J. Boston Soc. Civil Eng. 1943, 85, 219-237.

87. Pérez, J. S.; Porcel, E. R.; López, J. C.; Sevilla, J. F.; Chisti, Y. "Shear rate in stirred tank and bubble column bioreactors", Chem. Eng. J. 2006, 124, 1-5.

88. Pedocchi, F.; Piedra-Cueva, I. "Camp and stein's velocity gradient formalization", J. Environ. Eng. 2005, 131, 1369-1376.

89. Clark, M. M. "Critique of camp and stein's rms velocity gradient", J. Environ. Eng. 1985, 111, $741-754$.

90. Saatçi, A.; Halilsoy, M. "Discussion of 'critique of camp and stein's rms velocity gradient'by mm clark", $J$. Environ. Eng. 1986, 113, 675-678.

91. Kramer, T. A.; Clark, M. M. "Influence of strain-rate on coagulation kinetics", J. Environ. Eng. 1997, 123, 444-452.

92. Graber, S. D. "Discussion: Influence of strain-rate on coagulation kinetics", J. Environ. Eng. 1998, 124, 1028-1029.

93. Cleasby, J. L. "Is velocity gradient a valid turbulent flocculation parameter?", J. Environ. Eng. 1984, 110, 875-897.

94. Batchelor, G. K. "Mass transfer from small particles suspended in turbulent fluid", J. Fluid. Mech. 1980, 98, 609-623.

95. Saffman, P.; Turner, J. "On the collision of drops in turbulent clouds", J. Fluid. Mech. 1956, 1, 16-30.

96. Spielman, L. A. "Hydrodynamic Sspects of Flocculation", In The Scientific Basis of Flocculation; Ives, K.J., Eds.; Springer: New York, 1978; pp 63-88.

97. Argaman, Y.; Kaufman, W. J. "Turbulence on Orthokinetic Flocculation", In Serl Report; University of California, 1968.

98. Delichatsios, M. A.; Probstein, R. F. "Coagulation in turbulent flow: Theory and experiment", J. Colloid. Interf. Sci. 1975, 51, 394-405.

99. Mei, R.; Hu, K. C. "On the collision rate of small particles in turbulent flows", J. Fluid. Mech. 1999, 391, 67-89.

100. Lawler, D. F. "Removing particles in the water and wastewater", Envir. Sci. Tech. 1986, 20, 856-861.

101. Meyer, C.; Deglon, D. "Particle collision modeling-a review", Miner. Eng. 2011, 24, 719-730.

102. Zaccone, A.; Wu, H.; Lattuada, M.; Morbidelli, M. "Charged molecular films on brownian particles: Structure, interactions, and relation to stability", J. Phys. Chem. B 2008, 112, 6793-6802.

103. Swift, D. L.; Friedlander, S. K. "The coagulation of hydrosols by brownian motion and laminar shear flow", J. Coll. Sci. 1964, 19, 621-647.

104. Chang, H. N.; Robertson, C. R. "Platelet aggregation by laminar shear and brownian motion", Ann. Biomed. Eng. 1976, 4, 151-183.

105. Han, M.; Lawler, D. F. "The (relative) insignificance of g in flocculation", J. Amer. Water Works Assoc. 1992, 79-91.

106. Laurenzi, I. J.; Diamond, S. L. "Bidisperse aggregation and gel formation via simultaneous convection and diffusion", Ind. Eng. Chem. Res. 2002, 41, 413-420.

107. Van de Ven, T. G. Colloidal hydrodynamics; Academic Press: New York, 1989.

108. Van de Ven, T.; Mason, S. "The microrheology of colloidal dispersions vii. Orthokinetic doublet formation of spheres", Colloid. Polym. Sci. 1977, 255, 468-479.

109. Van de Ven, T.; Mason, S. "The microrheology of colloidal dispersions", Colloid. Polym. Sci. 1977, 255, 794-804.

110. Sheng-Hua, X.; Zhi-Wei, S.; Xu, L.; Wang, J. T. "Coupling effect of brownian motion and laminar shear 
flow on colloid coagulation: A brownian dynamics simulation study", Chinese Phys. B 2012, 21, 054702.

111. Van de Ven, T.; Mason, S. "The microrheology of colloidal dispersions: IV. Pairs of interacting spheres in shear flow", J. Colloid. Interf. Sci. 1976, 57, 505-516.

112. Pnueli, D.; Gutfinger, C.; Fichman, M. "A turbulent-brownian model for aerosol coagulation", Aerosol. Sci. Tech. 1991, 14, 201-209.

113. Zeichner, G.; Schowalter, W. "Use of trajectory analysis to study stability of colloidal dispersions in flow fields", AIChE J. 1977, 23, 243-254.

114. Zaccone, A.; Wu, H.; Gentili, D.; Morbidelli, M. "Theory of activated-rate processes under shear with application to shear-induced aggregation of colloids", Phys. Rev. E. 2009, 80, 051404.

115. Mohammadi, M.; Larson, E. D.; Liu, J.; Larson, R. G. "Brownian dynamics simulations of coagulation of dilute uniform and anisotropic particles under shear flow spanning low to high peclet numbers", J. Chem. Phys. 2015, 142, 024108.

116. Moritz, H. U. "Increase in viscosity and its influence on polymerization processes", Chem. Eng. Tech. 1989, 12, 71-87.

117. Bird, R. B.; Armstrong, R. C.; Hassager, O. Dynamics of Polymeric Liquids. Vol. 1: Fluid Mechanics; Wiley-Interscience: New York, 1987.

118. Larson, R. G. The Structure and Rheology of Complex Fluids; Oxford University Press: New York, 1999.

119. Kolhapure, N.; Fox, R. "CFD in polymer reaction engineering: Combining polymerization chemistry and detailed flow models", Dechema Monographien 2001, 247-272.

120. Wells, G.; Ray, W. "Investigation of imperfect mixing effects in the ldpe autoclave reactor using CFD and compartment models", Dechema Monographien 2001, 49-60.

121. Woods, M. E.; Krieger, I. M. "Rheological studies on dispersions of uniform colloidal spheres I. Aqueous dispersions in steady shear flow", J. Colloid. Interf. Sci. 1970, 34, 91-99.

122. Alexopoulos, A.; Maggioris, D.; Kiparissides, C. "Cfd analysis of turbulence non-homogeneity in mixing vessels: A two-compartment model", Chem. Eng. Sci. 2002, 57, 1735-1752.

123. Pohn, J.; Cunningham, M.; McKenna, T. F. "Scale- up of emulsion polymerization reactors part I-development of a model framework", Macromol. React. Eng. 2013, 7, 380-392.

124. Pohn, J.; Cunningham, M.; McKenna, T. F. "Scale- up of emulsion polymerization reactors part II-simulations and interpretations", Macromol. React. Eng. 2013, 7, 393-408.

125. Bos, M. T.; van Opheusden, J. H. "Brownian dynamics simulation of gelation and aging in interacting colloidal systems", Phys. Rev. E. 1996, 53, 5044.

126. Puertas, A.; Fernandez-Barbero, A.; de las Nieves, F. "Brownian dynamics simulation of diffusive mesoscopic particle aggregation", Compt. Phys. Commun. 1999, 121, 353-357.

127. Puertas, A.; Maroto, J.; Barbero, A. F.; De Las Nieves, F. "Particle interactions in colloidal aggregation by brownian dynamics simulation", Phys. Rev. E. 1999, 59, 1943-1947.

128. Markutsya, S.; Subramaniam, S.; Vigil, R. D.; Fox, R. O. "On brownian dynamics simulation of nanoparticle aggregation", Ind. Eng. Chem. Res. 2008, 47, 3338-3345.

129. Liu, J.; Larson, R. G. "Brownian dynamics method for simulation of binding kinetics of patterned colloidal spheres with hydrodynamic interactions", J. Chem. Phys. 2013, 138, 174904.

130. Zaccone, A.; Gentili, D.; Wu, H.; Morbidelli, M. "Shear-induced reaction-limited aggregation kinetics of brownian particles at arbitrary concentrations", J. Chem. Phys. 2010, 132, 134903.

131. Hong, C.-W. "From long-range interaction to solid-body contact between colloidal surfaces during forming", J. Eur. Ceram. Soc. 1998, 18, 2159-2167.

132. Kroupa, M.; Vonka, M.; Kosek, J. "Modeling the mechanism of coagulum formation in dispersions", 
Langmuir 2014, 30, 2693-2702.

133. Johnson, K.; Kendall, K.; Roberts, A. "Surface Energy and the Contact of Elastic Solids", In Proceedings of the Royal Society of London A: Mathematical, Physical and Engineering Sciences; The Royal Society, 1971; pp 301-313.

134. Kroupa, M.; Vonka, M.; Soos, M.; Kosek, J. "Size and structure of clusters formed by shear induced coagulation: Modeling by discrete element method", Langmuir 2015, 31, 7727-7737.

135. Kroupa, M.; Vonka, M.; Soos, M.; Kosek, J. "Utilizing the discrete element method for the modeling of viscosity in concentrated suspensions", Langmuir 2016, 32, 8451-8460.

136. Kemmere, M.; Meuldijk, J.; Drinkenburg, A.; German, A. "Aspects of coagulation during emulsion polymerization of styrene and vinyl acetate", J. Appl. Polym. Sci. 1998, 69, 2409-2421.

137. Kemmere, M.; Meuldijk, J.; Drinkenburg, A.; German, A. "Colloidal stability of high- solids polystyrene and polyvinyl acetate latices", J. Appl. Polym. Sci. 1999, 74, 1780-1791.

138. Sajjadi, S. "Particle formation and coagulation in the seeded semibatch emulsion polymerization of butyl acrylate", J. Polym. Sci. A1. 2000, 38, 3612-3630.

139. Pourmehr, M.; Navarchian, A. H. "Batch emulsion polymerization of vinyl chloride: Application of experimental design to investigate the effects of operating variables on particle size and particle size distribution", J. Appl. Polym. Sci. 2009, 111, 338-347.

140. Lazzari, S.; Jaquet, B.; Wu, H.; Morbidelli, M. "Shear stability of inverse latexes during their polymerization process", AIChE J. 2015, 61, 1380-1384.

141. Liu, B.; Wang, Y.; Zhang, M.; Zhang, H. "Initiator systems effect on particle coagulation and particle size distribution in one-step emulsion polymerization of styrene", Polymers 2016, 8, 55.

142. Šn̆upárek, J. "Particle coagulation at semicontinuous emulstion polymerization. I. Some factors affecting the process", J. Appl. Polym. Sci. 1979, 24, 909-914.

143. Zubitur, M.; Asua, J. "Factors affecting kinetics and coagulum formation during the emulsion copolymerization of styrene/butyl acrylate", Polymer 2001, 42, 5979-5985.

144. Zubitur, M.; Asua, J. M. "Agitation effects in the semicontinuous emulsion polymerization of styrene and butyl acrylate", J. Appl. Polym. Sci. 2001, 80, 841-851.

145. Hidalgo-Alvarez, R.; Martin, A.; Fernandez, A.; Bastos, D.; Martinez, F.; De Las Nieves, F. "Electrokinetic properties, colloidal stability and aggregation kinetics of polymer colloids", Adv. Colloid Interf. Sci. 1996, $67,1-118$.

146. Jódar-Reyes, A.; Ortega-Vinuesa, J.; Martín-Rodríguez, A. "Electrokinetic behavior and colloidal stability of polystyrene latex coated with ionic surfactants", J. Colloid. Interf. Sci. 2006, 297, 170-181.

147. Romero-Cano, M.; Martin-Rodriguez, A.; De Las Nieves, F. "Electrosteric stabilization of polymer colloids with different functionality", Langmuir 2001, 17, 3505-3511.

148. Hanus, L. H.; Hartzler, R. U.; Wagner, N. J. "Electrolyte-induced aggregation of acrylic latex. 1. Dilute particle concentrations", Langmuir 2001, 17, 3136-3147.

149. Sandkühler, P.; Sefcik, J.; Morbidelli, M. "Kinetics of aggregation and gel formation in concentrated polystyrene colloids", J. Phys. Chem. B 2004, 108, 20105-20121.

150. Reynhout, X.; Hoekstra, L.; Meuldijk, J.; Drinkenburg, A. "Contribution of steric and electrostatic repulsion forces to the stability of styrene latices copolymerized with acrylic acid", J. Polym. Sci. A1. 2003, 41, 2985-2995.

151. Reynhout, X. E.; Beckers, M.; Meuldijk, J.; Drinkenburg, B. A. "Electrosteric stability of styrene/acrylic acid copolymer latices under emulsion polymerization reaction conditions", J. Polym. Sci. A1. 2005, 43, 726-732. 
152. Soos, M.; Moussa, A. S.; Ehrl, L.; Sefcik, J.; Wu, H.; Morbidelli, M. "Effect of shear rate on aggregate size and morphology investigated under turbulent conditions in stirred tank", J. Colloid. Interf. Sci. 2008, 319, 577-589.

153. Méndez- Ecoscia, A. C.; Prates- Pereira, A. F.; Fonseca- Marques, M. B.; Sheibat- Othman, N.; McKenna, T. F. "A new methodology for measuring the stability of emulsion polymer particles", Macromol. Symp. 2016, 360, 142-151.

154. Ghosh, A. K.; Lindt, J. T. "Processing of polymer latex emulsion: Batch coagulation", J. Appl. Polym. Sci. 1990, 39, 1553-1568.

155. Torres, F. E.; Russel, W. B.; Schowalter, W. R. "Floc structure and growth kinetics for rapid shear coagulation of polystyrene colloids", J. Colloid. Interf. Sci. 1991, 142, 554-574.

156. Spicer, P. T.; Keller, W.; Pratsinis, S. E. "The effect of impeller type on floc size and structure during shear-induced flocculation", J. Colloid. Interf. Sci. 1996, 184, 112-122.

157. Selomulya, C.; Bushell, G.; Amal, R.; Waite, T. "Aggregation mechanisms of latex of different particle sizes in a controlled shear environment", Langmuir 2002, 18, 1974-1984.

158. Wu, H.; Zaccone, A.; Tsoutsoura, A.; Lattuada, M.; Morbidelli, M. "High shear-induced gelation of charge-stabilized colloids in a microchannel without adding electrolytes", Langmuir 2009, 25, 4715-4723.

159. Xie, D.; Wu, H.; Zaccone, A.; Braun, L.; Chen, H.; Morbidelli, M. "Criticality for shear-induced gelation of charge-stabilized colloids", Soft Matter 2010, 6, 2692-2698.

160. Zaccone, A.; Gentili, D.; Wu, H.; Morbidelli, M.; Del Gado, E. "Shear-driven solidification of dilute colloidal suspensions", Phys. Rev. Lett. 2011, 106, 138301.

161. Xie, D.; Qiao, G. G.; Dunstan, D. E. "Flow-induced aggregation of colloidal particles in viscoelastic fluids", Phys. Rev. E. 2016, 94, 022610.

162. Meng, X.; Wu, H.; Morbidelli, M. "Shear-driven aggregation of binary colloids for randomly distributing nanoparticles in a matrix", Soft Matter 2016, 12, 3696-3702.

163. Urrutia, J.; Peña, A.; Asua, J. M. "Reactor fouling by preformed latexes", Macromol. React. Eng. 2017, 11, $1-14$.

164. Jaquet, B.; Lazzari, S.; Colonna, L.; Colombo, G.; Soos, M.; Morbidelli, M. "Effects of coalescence on shear-induced gelation of colloids", Langmuir 2017, 33, 1180-1188.

165. Ishii, H.; Ishii, M.; Nagao, D.; Konno, M. "Advanced synthesis for monodisperse polymer nanoparticles in aqueous media with sub-millimolar surfactants", Polymer 2014, 55, 2772-2779.

166. Goodwin, J. W.; Ottewill, R. H.; Pelton, R.; Vianello, G.; Yates, D. E. "Control of particle size in the formation of polymer latices", Polym. Int. 1978, 10, 173-180.

167. Ugelstad, J.; Berge, A.; Ellingsen, T.; Schmid, R.; Nilsen, T.-N.; Mørk, P.; Stenstad, P.; Hornes, E.; Olsvik, Ø. "Preparation and application of new monosized polymer particles", Prog. Polym. Sci. 1992, 17, 87-161.

168. Ramimoghadam, D.; Bagheri, S.; Hamid, S. B. A. "Stable monodisperse nanomagnetic colloidal suspensions: An overview", Colloid. Surface. B 2015, 133, 388-411.

169. Killops, K. L.; Rodriguez, C. G.; Lundberg, P.; Hawker, C. J.; Lynd, N. A. "A synthetic strategy for the preparation of sub-100 nm functional polymer particles of uniform diameter", Polym. Chem. 2015, 6, 1431-1435.

170. Vanderhoff, J.; El-Aasser, M.; Micale, F.; Sudol, E.; Tseng, C.; Silwanowicz, A.; Kornfeld, D.; Vicente, F. "Preparation of large-particle-size monodisperse latexes in space: Polymerization kinetics and process development", J. Disper. Sci. Technol. 1984, 5, 231-246.

171. Ober, C. K.; Hair, M. L. "The effect of temperature and initiator levels on the dispersion polymerization of polystyrene", J. Polym. Sci. A1. 1987, 25, 1395-1407. 
172. Goodwin, J.; Hearn, J.; Ho, C.; Ottewill, R. "Studies on the preparation and characterisation of monodisperse polystyrene laticee", Colloid. Polym. Sci. 1974, 252, 464-471.

173. Shibuya, K.; Nagao, D.; Ishii, H.; Konno, M. "Advanced soap-free emulsion polymerization for highly pure, micron-sized, monodisperse polymer particles", Polymer 2014, 55, 535-539.

174. Goodall, A.; Wilkinson, M.; Hearn, J. "Mechanism of emulsion polymerization of styrene in soap- free systems", J. Polym. Sci. A1. 1977, 15, 2193-2218.

175. Reese, C. E.; Asher, S. A. "Emulsifier-free emulsion polymerization produces highly charged, monodisperse particles for near infrared photonic crystals", J. Colloid. Interf. Sci. 2002, 248, 41-46.

176. Xu, J.; Li, P.; Wu, C. "Formation of highly monodispersed emulsifier- free cationic poly (methylstyrene) latex particles", J. Polym. Sci. A1. 1999, 37, 2069-2074.

177. Aslamazova, T. "Emulsifier-free latexes and polymers on their base", Prog. Org. Coat. 1995, 25, 109-167.

178. Peng, B.; Imhof, A. "Surface morphology control of cross-linked polymer particles via dispersion polymerization", Soft Matter 2015, 11, 3589-3598.

179. Tamai, H.; Fujii, A.; Suzawa, T. "Some colloidal considerations on the surface characteristics of various emulsifier-free polymer latices", J. Colloid. Interf. Sci. 1987, 116, 37-41.

180. Okubo, M.; Kondo, Y.; Takahashi, M. "Production of submicron-size monodisperse polymer particles having aldehyde groups by seeded aldol condensation polymerization", Colloid. Polym. Sci. 1993, 271, 109-113.

181. Cao, K.; Yu, J.; Li, B.-G.; Li, B.-F.; Pan, Z.-R. "Micron-size uniform poly (methyl methacrylate) particles by dispersion polymerization in polar media: 1. Particle size and particle size distribution", Chem. Eng. J. 2000, 78, 211-215.

182. Gu, S.; Inukai, S.; Konno, M. "Preparation of monodisperse, micron-sized polystyrene particles with an amphoteric initiator in soapfree polymerization", J. Chem. Eng. Japan 2002, 35, 977-981.

183. Gu, S.; Mogi, T.; Konno, M. "Preparation of monodisperse, micron-sized polystyrene particles with single-stage polymerization in aqueous media", J. Colloid. Interf. Sci. 1998, 207, 113-118.

184. Boutti, S.; Zafra, R. D.; Graillat, C.; McKenna, T. F. "Interaction of surfactant and initiator types in emulsion polymerisations: A comparison of ammonium persulfate and hydrogen peroxide", Macromol. Chem. Phys. 2005, 206, 1355-1372.

185. Tuin, G.; Peters, A.; Van Diemen, A.; Stein, H. "Preparation of large monodisperse polystyrene particles by a one step surfactant-free emulsion polymerization", J. Colloid. Interf. Sci. 1993, 158, 508-510.

186. Ugelstad, J.; Mfutakamba, H.; Mørk, P.; Ellingsen, T.; Berge, A.; Schmid, R.; Holm, L.; Jørgedal, A.; Hansen, F.; Nustad, K. "Preparation and application of monodisperse polymer particles", J. Polym. Sci. Polym. Symp.1985, 225-240.

187. Essadam, H.; Pichot, C.; Guyot, A. "Narrow size distribution polystyrene latexes prepared in the presence of amphoteric sulfobetaine surfactants", Colloid. Polym. Sci. 1988, 266, 462-469.

188. Liu, B.; Zhang, M.; Zhou, C.; Ren, L.; Cheng, H.; Ao, Y.; Zhang, H. "Synthesis of monodisperse, large scale and high solid content latexes of poly (n-butyl acrylate) by a one-step batch emulsion polymerization", Colloid. Polym. Sci. 2013, 291, 2385-2398.

189. Cowell, C.; Vincent, B. "Flocculation kinetics and equilibria in sterically stabilized dispersions", J. Colloid. Interf. Sci. 1983, 95, 573-582.

190. Stenkamp, V. S.; Berg, J. C. "The role of long tails in steric stabilization and hydrodynamic layer thickness", Langmuir 1997, 13, 3827-3832.

191. Leckband, D.; Israelachvili, J. "Intermolecular forces in biology", Q. Rev. Biophys. 2001, 34, $105-267$.

192. Kemmere, M.; Meuldijk, J.; Drinkenburg, A.; German, A. "Rheology and flow during high solids emulsion 
polymerization of styrene", Polym. React. Eng. 1998, 6, 243-268.

193. Vasudevan, M.; Buse, E.; Lu, D.; Krishna, H.; Kalyanaraman, R.; Shen, A. Q.; Khomami, B.; Sureshkumar, R. "Irreversible nanogel formation in surfactant solutions by microporous flow", Nat. Mater. 2010, 9 , 436-441.

194. Mewis, J.; Wagner, N. J. "Current trends in suspension rheology", J. Non-newton. Fluid Mech. 2009, 157, 147-150.

195. Mackay, M. E.; Dao, T. T.; Tuteja, A.; Ho, D. L.; Van Horn, B.; Kim, H.-C.; Hawker, C. J. "Nanoscale effects leading to non-einstein-like decrease in viscosity", Nat. Mater. 2003, 2, 762-766.

196. Cross, M. M. "Rheology of non-newtonian fluids: A new flow equation for pseudoplastic systems", J. Coll. Sci. 1965, 20, 417-437.

197. Riddle, M. J.; Narvaez, C.; Bird, R. B. "Interactions between two spheres falling along their line of centers in a viscoelastic fluid", J. Non-newton. Fluid Mech. 1977, 2, 23-35.

198. Bot, E.; Hulsen, M.; Van den Brule, B. "The motion of two spheres falling along their line of centres in a boger fluid", J. Non-newton. Fluid Mech. 1998, 79, 191-212.

199. Snijkers, F.; Pasquino, R.; Vermant, J. "Hydrodynamic interactions between two equally sized spheres in viscoelastic fluids in shear flow", Langmuir 2013, 29, 5701-5713.

200. Scirocco, R.; Vermant, J.; Mewis, J. "Effect of the viscoelasticity of the suspending fluid on structure formation in suspensions", J. Non-newton. Fluid Mech. 2004, 117, 183-192.

201. Won, D.; Kim, C. "Alignment and aggregation of spherical particles in viscoelastic fluid under shear flow", J. Non-newton. Fluid Mech. 2004, 117, 141-146.

202. Guery, J.; Bertrand, E.; Rouzeau, C.; Levitz, P.; Weitz, D.; Bibette, J. "Irreversible shear-activated aggregation in non-brownian suspensions", Phys. Rev. Lett. 2006, 96, 198301.

203. Hamilton-Brown, P.; Bekard, I.; Ducker, W. A.; Dunstan, D. E. "How does shear affect a $\beta$ fibrillogenesis?", J. Phys. Chem. B 2008, 112, 16249-16252. 